\title{
Self-force on charges in the spacetime of spherical shells
}

\author{
Lior M. Burko and Yuk Tung Liu \\ Theoretical Astrophysics, California Institute of Technology, Pasadena, California 91125 \\ Yoav Soen \\ Department of Physics, Technion-Israel Institute of Technology, 32000 Haifa, Israel
}

(Received 27 August 2000; published 27 December 2000)

\begin{abstract}
We study the self-force acting on static electric or scalar charges inside or outside a spherical, massive, thin shell. The regularization of the self-force is done using the recently proposed mode sum regularization prescription. In all cases the self-force acting on the charge is repulsive. We find that in the scalar case the force is quadratic in the mass of the shell, and is a second post-Newtonian effect. For the electric case the force is linear in the shell's mass, and is a first post-Newtonian effect. When the charge is outside the shell our results correct the known zero self-force in the scalar case or the known repulsive, inverse-cubic force law in the electric case, for the finite size of the shell. When the charge is near the center of the shell the charge undergoes harmonic oscillations.
\end{abstract}

DOI: 10.1103/PhysRevD.63.024015

PACS number(s): 04.25.-g

\section{INTRODUCTION AND OVERVIEW}

The calculation of the self-force acting on a particle in curved spacetime has become recently exceedingly important, as the Laser Interferometer Space Antenna (LISA) is currently planned to fly as early as 2010. One of the most promising gravitational-wave sources for LISA is extreme mass-ratio binaries, where the evolution in the last year of inspiral is strongly affected by the self-force acting on the inspiraling object. The long-term goal then is to compute the momentary self-forces acting on a compact object, which is in a generic orbit around a supermassive black hole. Once the self-force is known, this force could be included in the determination of the orbital evolution under radiation reaction.

However, much is yet to be understood about the nature of self-forces in curved spacetime even for problems which are significantly simpler than the astrophysically motivated ones. These problems, nevertheless, are motivated by being pedagogical on the one hand, and by illuminating some important points of principle, which are relevant also for astrophysically realistic problems, on the other hand. A number of simple static configurations have thus been analyzed, including the self-force acting on scalar or electric charges held static in the spacetime of a Schwarzschild black hole [1-4], electric or magnetic dipoles which are static outside a Schwarzschild black hole [5], a static electric charge outside a Kerr black hole [6] or a Kerr-Newman black hole [7], a static electric charge in a spherically-symmetric Brans-Dicke field [8], and a static charge in the spacetime of a cosmic string $[9,10]$. In all these analyses the self-force was found to leading order in the charge of the particle, i.e., to order

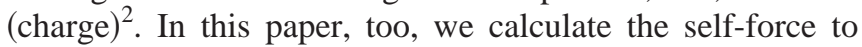
that order.

Still, there are elementary configurations where the selfforce acting on charges is as yet poorly understood. Specifically, little is known on the self-force acting on charges in the spacetime of spherical, massive (thin) shells. This problem is interesting from a pedagogical point of view because the derivation of the self-force is simple, whether the charge is inside the shell (where spacetime is locally flat) or outside the shell (where spacetime is locally Schwarzschild). However, despite the simplicity, there are some non-trivial issues which are demonstrated already in this simple context. First, when the charge is inside the shell, there is a non-zero selfforce acting on it, even when the charge is static. Second, this non-zero force exists although the charge moves along a geodesic of spacetime (a static worldline is a geodesic in flat spacetime). Third, already in this simple context it is evident that the Einstein equivalence principle is not satisfied by problems which involve self interaction. Namely, two identical static particles, one in a globally-Minkowski spacetime and one in a locally Minkowski spacetime experience different forces: the former a zero self-force and the latter a nonzero self-force, although the geometries of the local neighborhoods of the two particles are exactly identical. [The selfforce is considered here to order $(\text { charge })^{2}$. Under this assumption, the geometry is a fixed background, and is given by the solution of the Einstein equations in the absence of the charge. When higher order terms, of order (charge) ${ }^{4}$ or higher, are considered, the geometries in the local neighborhoods of the particles would no longer be identical, because of the different ways in which the particles' fields couple to the geometry in the two cases.] Because the self-force couples to the charge of the particle in a way which depends on the type of charge, the worldline of a particle which carries one type of charge deviates from the worldline of a particle which carries a different type of charge. The reason for this failure of the equivalence principle is obvious: the equivalence principle relates to the local neighborhood of the particle. The self-force, however, is affected by the boundary conditions on the surface of discontinuity (the shell), and these far-field conditions affect the near-field of the particle such that a self-force arises. In problems where there is nontrivial dynamics, this effect comes about by the scattering of the tails of the field off the spacetime curvature, and this scattering occurs also at arbitrary large distances from the position of the particle in the remote past. In this sense the self force is a non-local effect, which transcends the domain 
of applicability of the equivalence principle. The equivalence principle is transcended also when the particle is outside the spherical shell. Although spacetime outside a spherical shell of mass $M$ is identical to the spacetime outside a black hole of the same mass $M$ (by virtue of Birkhoff's theorem), the self-forces acting on two identical particles, one in a globally-Schwarzschild spacetime and the other in a locallySchwarzschild spacetime, are different. In principle, one could infer on (classes of) spherical mass distributions by solving the inverse problem, and finding the spherical mass distribution which induces a measured self-force. An important point of principle then, is that the interior of the source for the gravitational field is important for the orbital evolution of particles. As noted by Ryan [11], the orbital evolution under radiation reaction would enable us to map the spacetime around a black hole (or, possibly, some other dense objects). In addition, it would allow us also to infer on the interior of the source, its equation of state, its density distribution, etc.

The problem of the self force in the spacetime of a spherical massive shell was first considered by Unruh, who studied the case of a static electric charge $q$ inside a spherical shell of mass $M$ and radius $R$ [12]. Unruh found that there was a radial non-zero force acting on the charge. [The related problem of a point charge, coupled to a massive (Proca) vector field, within a spherical shell was studied by Léauté and Linet [13].] Below, we shall recapitulate Unruh's result, but derive it using the newly-proposed mode sum regularization prescription (MSRP) [14,15]. We shall also provide more insight into the meaning of the result. We find that the selfforce acting on the charge is directed toward the center of the sphere (the self force acts to repel the charge from the sphere). When the charge is very close to the center, this induces harmonic oscillations, with angular frequency of $\omega^{2}=\frac{1}{3}\left(G / c^{2}\right) q^{2} M /\left(m R^{4}\right)$, to leading order in $M / R$, where $m$ is the particle's mass. Here, $G$ is Newton's gravitational constant, and $c$ is the speed of light.

Then, we also study the self-force acting on an electric charge $q$ outside a spherical shell. For the case where the source of the gravitational field is a Schwarzschild black hole, the self-force was found by Smith and Will [2], who found that there was a repelling self-force, which was given by $f_{r}(r)=\left(G / c^{2}\right) q^{2} M / r^{3}$. Here, $q$ is the electric charge of the particle, $M$ is the black hole's mass, and $r$ is the usual radial Schwarzschild coordinate. (This expression for the force is exact in a reference frame of a freely-falling observer who is instantaneously at rest at the position of the charge.) When the source of the gravitational force is extended, the Smith-Will force is corrected by a finite-size term, which, to leading order in $M / r$, is of the same order in $G / c^{2}$ as the Smith-Will term. Specifically, we find that when the source is a thin spherical shell of mass $M$, to the leading order in $M / r$, the self-force is given by $f_{\hat{r}}=\left(G / c^{2}\right) q^{2} M / r^{3}[1$ $\left.+\frac{2}{3}(R / r)^{2}+O(R / r)^{4}\right]$, where $R$ is the radius of the shell. For $r \gg R$ (when the charge is very far from the shell) this correction is very small. However, when the charge is very close to the shell, higher-order terms in $(R / r)^{2}$ become comparable with the Smith-Will term, such that the correction terms may become more important than the leading-order term. We find that near the shell, the self-force becomes very large. (In fact, approaching the shell we find that the selfforce diverges. We shall study that effect in detail, and show that when the charge is very close to the shell our mathematical model breaks down, such that the physical self-force is always finite.)

Next, we calculate the self-force acting on a particle endowed with scalar charge $q$. For the case where the charge is inside the shell we again find a self-force which directs toward the center. However, this force is quadratic in $M / R$, and is of order $G^{2} / c^{4}$. When the charge is near the center, the self-force acting on it is again that of an harmonic oscillator, with angular frequency $\omega^{2}=\frac{1}{15}\left(G^{2} / c^{4}\right) q^{2} M^{2} /\left(m R^{5}\right)$, to the leading order in $M / R$. When the scalar charge is outside the spherical shell, we find a correction to the well known result of a zero self-force which is the case when the source for the gravitational field is a black hole $[1,3,4]$. To leading order in $M / r$, this self-force is given by $f_{\hat{r}}(r)$ $=\frac{1}{3}\left(G^{2} / c^{4}\right) q^{2}\left(M^{2} / r^{5}\right) R\left[1+O(R / r)^{2}\right]$. We find that when the source of the gravitational field is extended, there is a finite-size correction also for this case. However, because the zero-size force happens to vanish, the finite-size effect is always larger than the zero-size effect (unlike the electric charge case).

The regularization prescription we use in the calculation of the self-force is based on Ori's mode sum regularization prescription (MSRP) [14,15], which is an application of the axiomatic Quinn-Wald approach [16] and the approach of Mino, Sasaki, and Tanaka [17]. We note, that although the regularization procedures used in Refs. [5-9] yield the correct results for the regularized self-force, they are hard to generalize to cases where exact solutions are unknown, or where there is non-trivial dynamics. MSRP does not share this difficulty, and the regularization using MSRP is independent of the existence of an exact solution. Next, we describe MSRP very succinctly. More details are available in Refs. $[15,18]$. We note that MSRP has been developed in detail only for scalar charges, but it is likely that the approach is applicable in general also for higher-spin fields. The contribution to the physical self force from the tail part of the Green's function can be decomposed into stationary Teukolsky modes, and then summed over the frequencies $\omega$ and the azimuthal numbers $m$. The self-force equals then the limit $\epsilon \rightarrow 0^{-}$of the sum over all $l$ modes, of the difference between the force sourced by the entire worldline (the bare force ${ }^{\text {bare }} F_{\mu}^{l}$ ) and the force sourced by the half-infinite worldline to the future of $\epsilon$, where the particle has proper time $\tau=0$, and $\tau=\epsilon$ is an event along the past $(\tau<0)$ worldline. Next, we seek a regularization function $h_{\mu}^{l}$ which is independent of $\epsilon$, such that the series $\Sigma_{l}$ ( ${ }^{\text {bare }} F_{\mu}^{l}-h_{\mu}^{l}$ ) converges. Once such a function is found, the regularized selfforce is then given by ${ }^{\text {ren }} F_{\mu}=\Sigma_{l}\left({ }^{\text {bare }} F_{\mu}^{l}-h_{\mu}^{l}\right)-d_{\mu}$, where $d_{\mu}$ is a finite valued function. MSRP then shows, from a local integration of the Green's function, that the regularization function $h_{\mu}^{l}=a_{\mu} l+b_{\mu}+c_{\mu} l^{-1}$. For several cases, which have already been studied, MSRP yields the values of the functions $a_{\mu}, b_{\mu}, c_{\mu}$ and $d_{\mu}$ analytically. Alternatively, $a_{\mu}, b_{\mu}$, and $c_{\mu}$ (but not $d_{\mu}$ ) can also be found from the 
large- $l$ behavior of ${ }^{\text {bare }} F_{\mu}^{l}$. In addition to the (non-local) contribution of the tail's part of the Green's function to the self-force, there are also two additional, local terms: an Abraham-Lorentz-Dirac type term, and a term which couples to Ricci curvature and which preserves conformal invariance. For simple cases (including those we are interested in here) it was found that the finite term $d_{\mu}$ equals the sum of the two local contributions $[18,19]$, such that the total radiationreaction force can be found directly from the large- $l$ behavior of the modes of the bare force. MSRP has already been applied successfully for a number of cases: static scalar or electric charges in the spacetime of a Schwarzschild black hole [4], a scalar charge in uniform circular orbit around a Schwarzschild black hole [20], and a scalar charge plunging radially into a Schwarzschild black hole [21]. There is also strong evidence that MSRP is applicable also for electric charges $[22,4,19]$. Recently, a closely-related regularization procedure, based on Riemann $\zeta$-function regularization, was applied by Lousto for the case of a point mass falling radially into a Schwarzschild black hole [23]. Lousto considered an orbit which is geodesic in the absence of radiation-reaction effects, and computed the first-order correction of the spacetime metric and orbit. It is presently unclear, however, how to extend Lousto's method for non-geodesic orbits.

The organization of this paper is as follows. In Sec. II we study the self-force acting on a scalar charge inside (II A) and outside (II B) a spherical shell. In Sec. III we consider the self-force on an electric charge, both inside (III A) and outside (III B) the shell, and in Sec. IV make some heuristic comments regarding the physical origin of the self-force for the problem in question.

\section{SCALAR CHARGE}

Consider a thin uniform spherical shell of mass $M$ and radius $R$. In the spacetime of this shell we place a static particle. In this section the particle is endowed with a scalar

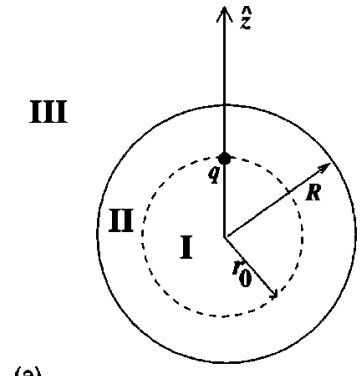

(a)

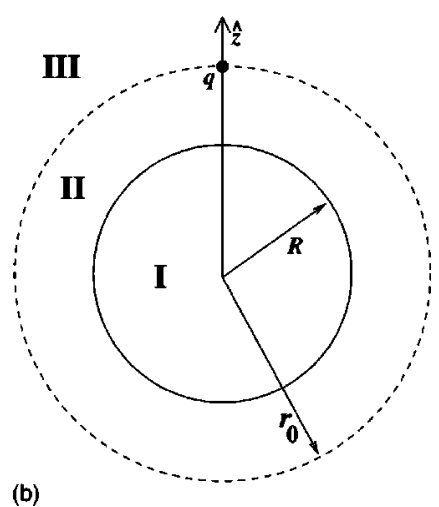

FIG. 1. A test charge (scalar or electric) $q$ is placed (a) inside and (b) outside a spherical shell of mass $M$ and radius $R$. In both cases the particle is at $r=r_{0}$, and without loss of generality the particle is positioned on the $\hat{z}$-axis. See the text for more details.

charge $q$. (In Sec. III we consider the case where the particle is electrically charged.) We consider the scalar field to be a linearized, test field in the geometry of the shell's spacetime. That is, the field is uncoupled to the geometry. This simplification allows us to obtain the self-force to order $q^{2}$. The particle is static at radius $r=r_{0}$, with either $r_{0}<R$ (in Sec. II A) or $r_{0}>R$ (in Sec. II B) (see Fig. 1). The fixed background geometry is described by the metric

$$
d s^{2}= \begin{cases}-\left(1-\frac{2 M}{R}\right) d t^{2}+d r^{2}+r^{2} d \theta^{2}+r^{2} \sin ^{2} \theta d \varphi^{2}, & r<R, \\ -\left(1-\frac{2 M}{r}\right) d t^{2}+\left(1-\frac{2 M}{r}\right)^{-1} d r^{2}+r^{2} d \theta^{2}+r^{2} \sin ^{2} \theta d \varphi^{2}, & r>R .\end{cases}
$$

Here the radial coordinate $r$ is defined such that the surface area of the 2-sphere $r=$ const, $t=$ const is $4 \pi r^{2}$, and $t$ is the (proper) time of a static observer at infinity. The spacetime (1) is Schwarzschild outside the shell and Minkowski inside the shell. Note that $g_{t t} \rightarrow-1$ as $r \rightarrow \infty$, but $g_{t t} \neq-1$ inside the shell, although spacetime is (locally) Minkowski.

The linearized field equation for a minimally coupled, massless scalar field $\Phi$ is given by

$$
\nabla_{\mu} \nabla^{\mu} \Phi\left(x^{\alpha}\right)=-4 \pi \rho\left(x^{\alpha}\right),
$$

where $\nabla_{\mu}$ denotes covariant differentiation compatible with the metric (1). The charge density $\rho$ is given by

$$
\rho\left(x^{\mu}\right)=q \int_{-\infty}^{\infty} d \tau \frac{\delta^{4}\left[x^{\mu}-z^{\mu}(\tau)\right]}{\sqrt{-g}} .
$$

Here $q$ is the total charge, $\tau$ is the proper time, $g$ is the metric determinant, and $z^{\mu}$ is the worldline of the charge. Without loss of generality, we place the charge on the $z$-axis at $r$ $=r_{0}$ and $\theta=0$. 


\section{A. Scalar charge inside a spherical shell}

\section{Derivation of the bare force}

In this section we shall study the case where the scalar charge is inside the shell, i.e., $r_{0}<R$. Then both $\rho$ and $\Phi$ can be decomposed into a sum over the Legendre polynomials according to

$$
\begin{aligned}
\rho(r, \theta) & =\frac{q \delta\left(r-r_{0}\right)}{4 \pi r_{0}^{2}} \sum_{l=0}^{\infty}(2 l+1) P_{l}(\cos \theta) \\
\Phi(r, \theta) & =\sum_{l=0}^{\infty} \phi^{l}(r) P_{l}(\cos \theta) .
\end{aligned}
$$

Note that because $d t / d \tau=1 / \sqrt{-g_{t t}}$, the factor $\sqrt{-g_{t t}}$ in the metric determinant $g$ is canceled. Also note that the series in Eq. (4) diverges. This is indeed what is expected, because the particle is construed as pointlike. Obviously, the charge density of a pointlike particle diverges on its worldline.

Substitution of Eqs. (4) and (5) into Eq. (2) yields

$$
\begin{aligned}
& \phi_{, r r}^{l}+\frac{2}{r} \phi_{, r}^{l}-\frac{l(l+1)}{r^{2}} \phi^{l}=-q \frac{2 l+1}{r_{0}^{2}} \delta\left(r-r_{0}\right), \quad r<R, \\
& \left(1-\frac{2 M}{r}\right) \phi_{, r r}^{l}+\frac{2}{r^{2}}(r-M) \phi_{, r}^{l}-\frac{l(l+1)}{r^{2}} \phi^{l}=0, \quad r>R,
\end{aligned}
$$

where commas denote partial derivatives. The solution for the inhomogeneous equation for $r<R$ is given by a linear combination of the solutions which solve the corresponding homogeneous equation, i.e., a linear combination of $r^{l}$ and $r^{-l-1}$. Outside the shell, where $r>R$, the solution is a linear combination of the Legendre functions $P_{l}(r / M-1)$ and $Q_{l}(r / M-1)$ [4]. Note that $P_{l}(z)$ diverges and $Q_{l}(z)$ vanishes as $z \rightarrow \infty$ for all values of $l$. [Except for $l=0$, as $P_{0}(z)=1$ for all values of $z$. However, as we require that all the individual modes of the field $\phi^{l}$ fall off at infinity $(r$ $\rightarrow \infty)$, this behavior of $P_{0}(z)$ is enough to rule it out as the relevant solution for large values of $r$.] We next require that $\phi^{l}(r)$ be regular both at infinity and at the origin. We thus write the solution for Eq. (6) as

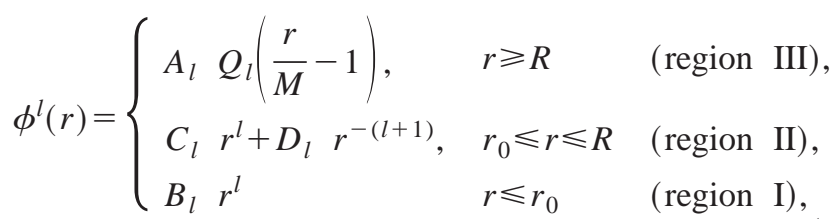

where $A_{l}, B_{l}, C_{l}$, and $D_{l}$ are constants to be determined by matching conditions. Specifically, we require that $\phi^{l}(r)$ be continuous everywhere (in particular, continuous also across $r=r_{0}$ and $r=R$ ).

Integrating Eq. (6) over $r$ from $r=r_{0}-\epsilon$ to $r=r_{0}+\epsilon$ and taking the limit $\epsilon \rightarrow 0^{+}$, and using the continuity of $\phi^{l}(r)$ across $r_{0}$, we find

$$
\lim _{\epsilon \rightarrow 0^{+}}\left[\phi_{, r}^{l}\left(r_{0}+\epsilon\right)-\phi_{, r}^{l}\left(r_{0}-\epsilon\right)\right]=-\frac{(2 l+1) q}{r_{0}^{2}} .
$$

The integration of Eq. (6) over $r$ from $r=R-\epsilon$ to $r=R+\epsilon$ is complicated by the discontinuity of $g_{r r}$ across $r=R$. This unnecessary complication can be removed by introducing the new radial coordinate $u(r)$, defined by

$$
u \equiv \begin{cases}r, & r \geqslant R, \\ \sqrt{1-\frac{2 M}{R}(r-R)+R,} & r \leqslant R .\end{cases}
$$

With this new radial coordinate, the metric (1) becomes

$$
d s^{2}=-K(u) d t^{2}+\frac{d u^{2}}{K(u)}+r(u)^{2}\left(d \theta^{2}+\sin ^{2} \theta d \varphi^{2}\right) .
$$

Here, $r$ is considered to be a function of $u$ obtained by the inverse transform of Eq. (9), and

$$
K(u)= \begin{cases}\left(1-\frac{2 M}{u}\right), & u \geqslant R, \\ \left(1-\frac{2 M}{R}\right), & u \leqslant R .\end{cases}
$$

In this new gauge, $g_{u u}$ is continuous everywhere (and so are the other metric coefficients) although the gradients of the metric functions $g_{t t, u}, g_{u u, u}$, and $r_{, u}$ are still discontinuous at $u=R$. In terms of $u$, Eq. (6) becomes

$$
K \phi_{, u u}^{l}+\left(\frac{2 K}{r} \frac{d r}{d u}+K_{, u}\right) \phi_{, u}^{l}-\frac{l(l+1)}{r^{2}} \phi^{l}=0 \quad \text { for } r \neq r_{0}
$$

Recall now that $\phi^{l}$ is continuous everywhere. However, $\phi_{, u}^{l}$ may at the most be step-function discontinuous at $u=R$ (the strongest form of discontinuity it may have is a step-function discontinuity; however, below we find that it is, in fact, continuous) such that $\phi_{\text {,u }}^{l}$ may, at the most, behave like a $\delta$-function (however, below we find that it is, in fact, proportional to a step-function discontinuity). In addition, also $K_{, u}$ and $r_{, u}$ are step-function discontinuous, but do not involve $\delta$-functions. Consequently, when we integrate Eq. (12) over $u$ from $u=R-\epsilon$ to $u=R+\epsilon$ and take the limit $\epsilon$ $\rightarrow 0^{+}$, only the contribution of the first term can be nonvanishing (because that is the only term which may involve a $\delta$-function), and is given, after integration by part, by

$$
\lim _{\epsilon \rightarrow 0^{+}}\left(\left.K \phi_{, u}^{l}\right|_{R-\epsilon} ^{R+\epsilon}-\int_{R-\epsilon}^{R+\epsilon} K_{, u} \phi_{, u}^{l} d u\right)=0
$$

The second term vanishes since it does not contain a $\delta$-function, which implies that $\phi_{, u}^{l}$ is continuous at $u=R$. In view of Eq. (9), this means that 


$$
\sqrt{1-\frac{2 M}{R}} \phi_{, r}^{l}\left(R^{+}\right)=\phi_{, r}^{l}\left(R^{-}\right)
$$

Here, $R^{ \pm}$denotes $\lim _{\epsilon \rightarrow 0^{+}}(R \pm \epsilon)$.

The constants $A_{l}, B_{l}, C_{l}$ and $D_{l}$ in (7) are determined by the matching conditions of $\phi^{l}$, which are (to reiterate): (1) $\phi^{l}$ is continuous everywhere; (2) $\phi^{l}$ vanishes at $r=\infty$ and is finite at $r=0$ [these two conditions have been taken into account in writing Eq. (7)]; (3) $\phi_{, r}^{l}$ is discontinuous at $r$ $=r_{0}$ and $r=R$ according to Eqs. (8) and (13). The results are

$$
\begin{aligned}
& A_{l}=q \frac{(2 l+1) \frac{M}{R}}{l \frac{M}{R} Q_{l}-\sqrt{1-\frac{2 M}{R}} Q_{l}^{\prime}} \frac{r_{0}^{l}}{R^{l+1}} \\
& C_{l}=-q \frac{r_{0}^{l}}{R^{2 l+1}} E_{l}^{s}
\end{aligned}
$$

$$
D_{l}=q r_{0}^{l}
$$

$$
B_{l}=\frac{q}{r_{0}^{l+1}}+C_{l}
$$

where $Q_{l}^{\prime}$ is the derivative of $Q_{l}$ with respect to its argument and the argument of both $Q_{l}$ and $Q_{l}^{\prime}$ is $(R / M)-1$, and

$$
E_{l}^{s}=\frac{\sqrt{1-\frac{2 M}{R} Q_{l}^{\prime}+(l+1) \frac{M}{R} Q_{l}}}{\sqrt{1-\frac{2 M}{R}} Q_{l}^{\prime}-l \frac{M}{R} Q_{l}} .
$$

Collecting our results, the field $\Phi$ is given by

$$
\Phi(r, \theta)=\left\{\begin{array}{lll}
\sum_{l=0}^{\infty} A_{l} Q_{l}\left(\frac{r}{M}-1\right) P_{l}(\cos \theta), & r \geqslant R & \text { (region III), } \\
\sum_{l=0}^{\infty} q \frac{r_{0}^{l}}{r^{l+1}} P_{l}(\cos \theta)-\sum_{l=0}^{\infty} \frac{q}{R}\left(\frac{r_{0}}{R}\right)^{l}\left(\frac{r}{R}\right)^{l} E_{l}^{s} P_{l}(\cos \theta), & r_{0} \leqslant r \leqslant R & \text { (region II), } \\
\sum_{l=0}^{\infty} q \frac{r^{l}}{r_{0}^{l+1}} P_{l}(\cos \theta)-\sum_{l=0}^{\infty} \frac{q}{R}\left(\frac{r_{0}}{R}\right)^{l}\left(\frac{r}{R}\right)^{l} E_{l}^{s} P_{l}(\cos \theta), & r \leqslant r_{0} & \text { (region I). }
\end{array}\right.
$$

We are interested in the self force acting on the charge, which results from the field in the neighborhood of the particle, i.e., the field around $r=r_{0}$. As $r_{0}<R$, the field in the neighborhood of the charge is given by

$$
\Phi(r<R, \theta)=\sum_{l=0}^{\infty} q\left[\frac{r^{l}}{r_{0}^{l+1}} \Theta\left(r_{0}-r\right)+\frac{r_{0}^{l}}{r^{l+1}} \Theta\left(r-r_{0}\right)\right] P_{l}(\cos \theta)-\sum_{l=0}^{\infty} \frac{q}{R}\left(\frac{r_{0}}{R}\right)^{l}\left(\frac{r}{R}\right)^{l} E_{l}^{s} P_{l}(\cos \theta)
$$

Here, $\Theta(x)$ is the Heaviside step function, i.e., $\Theta(x)=1$ if $x>0$ and $\Theta(x)=0$ if $x<0$. To find the self-force, we next calculate the force according to $f_{\mu}=q \nabla_{\mu} \Phi$, and evaluate this expression at the position of the particle, i.e., at $r=r_{0}$. [We note that the alternative force law which is frequently used, i.e., $f_{\mu}=q\left(\nabla_{\mu} \Phi+u_{\mu} u^{\nu} \nabla_{\nu} \Phi\right)$, is likely not to be derivable from an action principle without introduction of non-linear coupling terms [24]. The simpler force law we use was recently justified by Quinn [25] using stress-energy considerations. Notice, however, that for the static particle we assume here the two force laws coincide.] Clearly, only the radial component of the force $f_{\mu}$ is non-zero. The (bare) radial force is then given by

$$
f_{r}^{\text {bare }}=-\sum_{l=0}^{\infty}\left[\frac{q^{2}}{2 r_{0}^{2}}+\left(\frac{q}{R}\right)^{2} l E_{l}^{s}\left(\frac{r_{0}}{R}\right)^{2 l-1}\right]
$$

Clearly, $f_{r}^{\text {bare }}$ diverges.

\section{Regularization of the bare force}

In order to find the regularized, physical self-force we next use MSRP. First, we find the regularization function $h_{r}=a_{r} l+b_{r}+c_{r} l^{-1}$. Note that the functions $a_{r}, b_{r}$, and $c_{r}$ are found from a local analysis of the Green's function, in the neighborhood of the worldline. However in the neighborhood of the worldline spacetime is Minkowski. For a static 
scalar particle in Minkowski spacetime the values of $a_{r}, b_{r}$, and $c_{r}$ are known, and are given by $a_{r}=0=c_{r}$, and $b_{r}=$ $-q^{2} /\left(2 r_{0}^{2}\right)$. (Note that these values were obtained for the gauge where $g_{t t}=-1$. However, re-definition of the coordinate $t$ does not change the values of these functions. Another point to be made here is that we use here the "averaged" value for $a_{r}$, which vanishes. The analysis can also be carried out using the "one-sided" values $a_{r}^{ \pm}$, or any of their linear combinations. However, MSRP guarantees the same result for the regularized self-force whatever the choice for $a_{r}$ may be. For more details see Ref. [15].) Next, for a static scalar particle in Minkowski spacetime $d_{r}=0$, such that the regularized radial self-force is given by

$$
\begin{aligned}
f_{r} & =-\sum_{l=0}^{\infty}\left[\frac{q^{2}}{2 r_{0}^{2}}+\left(\frac{q}{R}\right)^{2} l E_{l}^{s}\left(\frac{r_{0}}{R}\right)^{2 l-1}+b_{r}\right] \\
& =-\left(\frac{q}{R}\right)^{2} \sum_{l=0}^{\infty} l E_{l}^{s}\left(\frac{r_{0}}{R}\right)^{2 l-1} .
\end{aligned}
$$

This expression for $f_{r}$ is guaranteed by MSRP to be finite, and also to be the correct, physical, total self-force.

\section{Properties of the regularized force}

It can be shown that $E_{l}^{s}$ is always positive, such that the self-force directs towards the origin. In terms of the physical forces present in the problem, this force arises from the sphere, and acts to repel the charge from it. To gain more insight into this result, we next expand $E_{l}^{s}$ in $M / R$. This expansion is given by

$$
E_{l}^{s}=\frac{l+1}{2(2 l+1)(2 l+3)}\left(\frac{M}{R}\right)^{2}+O\left(\frac{M}{R}\right)^{3},
$$

such that the self-force becomes

$$
\begin{aligned}
f_{r}= & -\left(\frac{q}{R}\right)^{2}\left(\frac{M}{R}\right)^{2} \\
& \times\left[\sum_{l=1}^{\infty} \frac{l(l+1)}{2(2 l+1)(2 l+3)}\left(\frac{r_{0}}{R}\right)^{2 l-1}+O\left(\frac{M}{R}\right)\right] .
\end{aligned}
$$

To the leading order in $M / R$ the self-force is given by

$$
f_{r}=-q^{2} \frac{G^{2}}{c^{4}} \frac{M^{2}}{R^{4}}\left[\frac{3-x_{0}^{2}}{16 x_{0}^{3}\left(1-x_{0}^{2}\right)}-\frac{3+x_{0}^{2}}{32 x_{0}^{4}} \ln \left(\frac{1+x_{0}}{1-x_{0}}\right)\right],
$$

where $x_{0} \equiv r_{0} / R$. In Eq. (22) we re-introduced Newton's gravitational constant $G$, and the speed of light $c$. Equation (22) implies that this force is a 2nd post-Newtonian effect.

In this small $M / R$ limit, we further consider two cases. The first case is the charge being close to the center. The self-force is

$$
f_{r}=-\frac{1}{15} q^{2} \frac{G^{2}}{c^{4}} \frac{M^{2}}{R^{5}} r_{0}\left[1+O\left(\frac{r_{0}}{R}\right)^{2}\right] .
$$

The charge then oscillates under this self-force at angular frequency

$$
\omega^{2}=\frac{1}{15} q^{2} \frac{G^{2}}{c^{4}} \frac{M^{2}}{m R^{5}},
$$

where $m$ is the particle's mass. We note that although the force law (23) corresponds to the case where the particle is static, when the deviation from the center is small enough, the velocity during the oscillations is also small such that the correction to the static force-law is negligible, and the damped oscillations' frequency is, to first order, unchanged.

The next case we consider is the case where the charge approaches the shell, i.e. $x_{0} \rightarrow 1^{-}$. To the leading orders in $1-x_{0}$ the self-force becomes

$$
\begin{aligned}
f_{r}= & -q^{2} \frac{G^{2}}{c^{4}} \frac{M^{2}}{R^{4}}\left\{\frac{1}{16\left(1-x_{0}\right)}+\frac{1}{8} \ln \left(1-x_{0}\right)+\frac{9}{32}-\frac{\ln 2}{8}\right. \\
& \left.+O\left[\left(1-x_{0}\right) \ln \left(1-x_{0}\right)\right]\right\},
\end{aligned}
$$

which implies that the self force increases when the particle is closer to the shell. In fact, in the limit $x_{0} \rightarrow 1^{-}$this selfforce diverges. Recall that this self-force is supposedly the regularized, physical self force, which should be everywhere finite, even at the surface of discontinuity. Below, in Sec. IV we discuss the origin for the self-force. We show there that this origin is the gravitational interaction between the charge's field and the shell's mass elements. (In fact, in Sec. IV the analysis is done to only linearized order in $M$, such that in the scalar case, where the force is quadratic in $M$, it yields a zero effect; however, for the electric case, where the self-force is linear in $M$, it yields a result in total agreement with our analysis below in Sec. III.) Due to the spherical symmetry of the shell, clearly only the parts of the field which are external to the shell contribute to the total interaction. The closer the particle to the shell, the stronger the field in the exterior of the shell in the neighborhood of the latter, such that indeed one expects the interaction to be stronger. The divergence we find in the coincidence limit is a shortcoming of the mathematical model we use. Recall that we treat both the shell and the particle as mathematical $\delta$-functions. In actuality, one should endow both with finite extensions, which would remove this divergence. The $\delta$-functions model fails because it is incompatible with the assumption of staticity which we make. Specifically, the pointlike particle has some finite energy density and mass. In the electric analog, the electrically-charged particle has an electrostatic field which gravitates, and a mass. For an electron the charge is much larger than the mass, such that the latter can be ignored for the purpose of this discussion. The gravitational effect of the electrostatic field acts then to repel the shell. Approaching the shell this repulsion grows unboundedly, such that at some finite distance the internal stresses in the shell will no longer hold, and the geometry becomes dynamic. Hence, the assumption of staticity fails in the coincidence limit. When the particle is endowed with a large mass (but still considered as pointlike), it is, in fact, a 


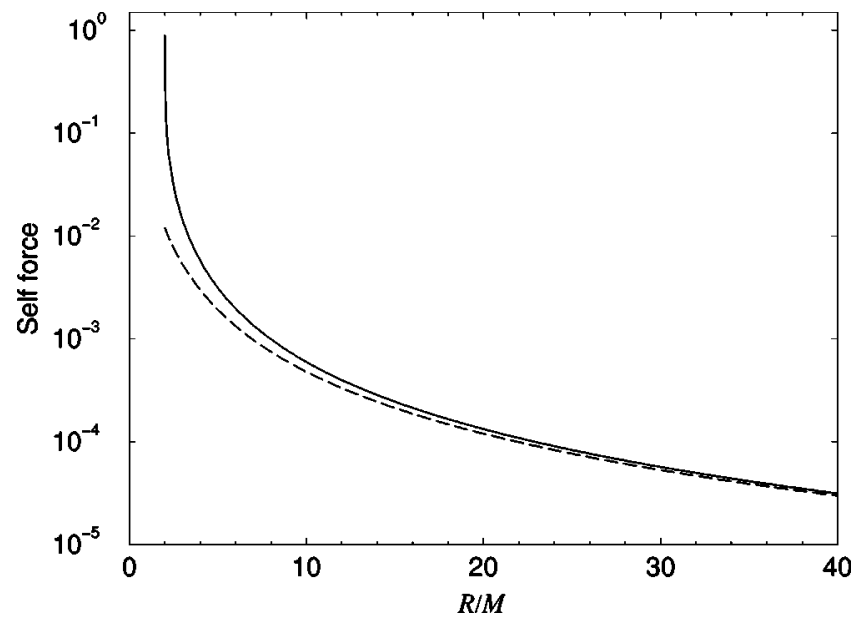

FIG. 2. The magnitude of the self-force $\left|f_{\hat{r}}\right|$, in units of $q^{2} G^{2} /\left(R^{2} c^{4}\right)$, experienced by a static, test, scalar charge as a function of $R / M$ computed by the full expression Eq. (19) (solid line) and the small $M / R$ expansion (22) (dashed line). The charge is placed inside the shell at $r_{0} / R=1 / 2$, and the self-force approaches the limit (8/9) $q^{2} G^{2} /\left(R^{2} c^{4}\right)$ predicted by Eq. (26) when $R / M \rightarrow 2$.

tiny black hole. When that black hole is put too close to the shell, the shell's internal stresses would no longer be able to hold against the pull of the particle's gravity, and the shell would break. In our mathematical analysis we assume that the entire configuration is static, and this assumption fails in the coincidence limit. An analogous discussion can be made also for a particle endowed with a scalar charge.

Next we consider the case when $M / R$ is not small. Figure 2 shows the magnitude of the self-force in units of $q^{2} G^{2} /\left(c^{4} R^{2}\right)$ as a function of $R / M$ calculated by the full expression (19) (solid line) and the small $M / R$ expression (22) (dashed line). (This full expression, and similarly the full expressions computed below, is computed using the numerical method outlined in Ref. [4].) The charge is placed at $r_{0} / R=1 / 2$. It can be seen that the self-force increases as $R / M$ decreases and the linear expression (22) deviates from the full expression. It can be shown that the self-force approaches

$$
f_{r} \rightarrow-\frac{q^{2}}{R^{2}} \frac{G^{2}}{c^{4}} \frac{x_{0}}{\left(1-x_{0}^{2}\right)^{2}}
$$

when $R / M \rightarrow 2$.

\section{Alternative regularization procedure}

We remark that the regularization procedure, which we performed using MSRP, can be justified by a more straightforward, albeit less robust approach. We can make direct use of the scalar-field comparison axiom of Quinn [25] (which is modeled after the comparison axiom for electric and gravitational fields of Quinn and Wald, for which plausibility arguments were given [16]). The scalar-field comparison axiom states the following (see [25] for more details): Consider two points, $P$ and $\widetilde{P}$, each lying on time-like worldlines in possibly different spacetimes which contain Klein-Gordon fields $\phi$ and $\widetilde{\phi}$ sourced by particles of charge $q$ on the worldlines. If the four-accelerations of the worldlines at $P$ and $\widetilde{P}$ have the same magnitude, and if we identify the neighborhoods of $P$ and $\widetilde{P}$ via the exponential map such that the four-velocities and four-accelerations are identified via Riemann normal coordinates, then the difference between the scalar forces $f_{\mu}$ and $\widetilde{f}_{\mu}$ is given by the limit $x \rightarrow 0$ of the field gradients, averaged over a sphere at geodesic distance $x$ from the worldline at $P$, i.e.,

$$
f_{\mu}-\tilde{f}_{\mu}=\lim _{x \rightarrow 0} q\left\langle\nabla_{\mu} \phi-\tilde{\nabla}_{\mu} \widetilde{\phi}\right\rangle_{x}
$$

We note that this axiom assumes a nearly trivial form for the case in question: the local neighborhood of the particle in question and of a similar particle in a (globally-)Minkowski spacetime are identical. (It is only the far-away properties of spacetime which are different for the two spacetimes.) Another remark is that we do not need to average here over directions, as the forces in our case are direction independent. Setting our minds to use this comparison axiom, we write the scalar field (16) as

$$
\Phi(r, \theta)= \begin{cases}\sum_{l=0}^{\infty} A_{l} Q_{l}\left(\frac{r}{M}-1\right) P_{l}(\cos \theta), & r \geqslant R, \\ \Phi_{c}(r, \theta)+\Delta \Phi_{s}(r, \theta), & r \leqslant R,\end{cases}
$$

where

$$
\begin{aligned}
\Phi_{c}(r, \theta) & =\sum_{l=0}^{\infty} q\left[\frac{r^{l}}{r_{0}^{l+1}} \Theta\left(r_{0}-r\right)+\frac{r_{0}^{l}}{r^{l+1}} \Theta\left(r-r_{0}\right)\right] P_{l}(\cos \theta) \\
& =\sum_{l=0}^{\infty} q \frac{r_{<}^{l}}{r_{>}^{l+1}} P_{l}(\cos \theta) \\
& =\frac{q}{\left|\boldsymbol{r}-r_{0} \hat{z}\right|}
\end{aligned}
$$

is just the usual scalar field for a particle at rest in a globallyMinkowski spacetime, and $\Delta \Phi_{s}(r, \theta)$ is a term which corrects for the finite size of the Minkowski patch of spacetime, which is given by

$$
\Delta \Phi_{s}(r, \theta)=-\sum_{l=0}^{\infty} \frac{q}{R}\left(\frac{r_{0}}{R}\right)^{l}\left(\frac{r}{R}\right)^{l} E_{l}^{s} P_{l}(\cos \theta) .
$$

Here $r_{<}=\min \left(r, r_{0}\right)$ and $r_{>}=\max \left(r, r_{0}\right)$. Hence inside the shell, the scalar field contains the Coulomb field $\Phi_{c}$ and a correction $\Delta \Phi_{s}$. We next identify $\Phi_{c}$ with $\widetilde{\phi}$. The self-force therefore arises only from $\Delta \Phi_{s}$, and is given by

$$
\begin{aligned}
f_{r} & =q\left(\Delta \Phi_{s}\right)_{, r} \\
& =-\left(\frac{q}{R}\right)^{2} \sum_{l=1}^{\infty} l E_{l}^{s}\left(\frac{r_{0}}{R}\right)^{2 l-1},
\end{aligned}
$$


a result which is identical to Eq. (19). (Alternatively, we can argue that the self-force on a static particle in a globallyMinkowski spacetime is zero, such that $\Phi_{c}$ does not contribute to the self-force. The self-force therefore arises only from $\left.\Delta \Phi_{s} \cdot\right)$

\section{B. Scalar charge outside the shell}

\section{Derivation of the bare force}

Next, we study the case where the scalar charge is outside the shell, i.e., the case where $r_{0}>R$. Now, the charge density (3) is decomposed as

$$
\rho(r, \theta)=\frac{q}{4 \pi} \frac{\delta\left(r-r_{0}\right)}{r_{0}^{2}} \sqrt{1-\frac{2 M}{r_{0}}} \sum_{l=0}^{\infty}(2 l+1) P_{l}(\cos \theta)
$$

[instead of Eq. (4)] and the potential is decomposed as in Eq. (5). Then the scalar-field equation $\nabla_{\mu} \nabla^{\mu} \Phi=-4 \pi \rho$ becomes

$$
\begin{gathered}
\left(1-\frac{2 M}{r}\right) \phi_{, r r}^{l}+\frac{2}{r^{2}}(r-M) \phi_{, r}^{l}-\frac{l(l+1)}{r^{2}} \phi_{l}=-q \frac{2 l+1}{r_{0}^{2}} \delta\left(r-r_{0}\right) \sqrt{1-\frac{2 M}{r_{0}}}, \quad r>R, \\
\phi_{, r r}^{l}+\frac{2}{r} \phi_{, r}^{l}-\frac{l(l+1)}{r^{2}} \phi_{l}=0, \quad r<R .
\end{gathered}
$$

Our solution here follows closely the solution of the preceding section.

The $\delta$-function in Eq. (34) gives one of the matching conditions:

$$
\lim _{\epsilon \rightarrow 0^{+}}\left[\phi_{, r}^{l}\left(r_{0}+\epsilon\right)-\phi_{, r}^{l}\left(r_{0}-\epsilon\right)\right]=-q \frac{2 l+1}{r_{0}^{2}}\left(1-\frac{2 M}{r_{0}}\right)^{-1 / 2}
$$

while the other matching conditions are the same as in the case when the scalar charge is inside the shell. The solution is expressed as a linear combination of Legendre functions $P_{l}$ and $Q_{l}$ with arguments $r / M-1$ for $r>R$, and is proportional to $r^{l}$ for $r<R$. Hence,

$$
\phi^{l}(r)=\left\{\begin{array}{lll}
A_{l} Q_{l}\left(\frac{r}{M}-1\right), & r \geqslant r_{0} & \text { (region III), } \\
C_{l} P_{l}\left(\frac{r}{M}-1\right)+D_{l} Q_{l}\left(\frac{r}{M}-1\right), & R \leqslant r \leqslant r_{0} & \text { (region II), } \\
B_{l} r^{l}, & r \leqslant R & \text { (region I), }
\end{array}\right.
$$

where the constants $A_{l}, B_{l}, C_{l}$ and $D_{l}$ are determined by the matching conditions. The result for the scalar field $\Phi(r, \theta)$ is then given by

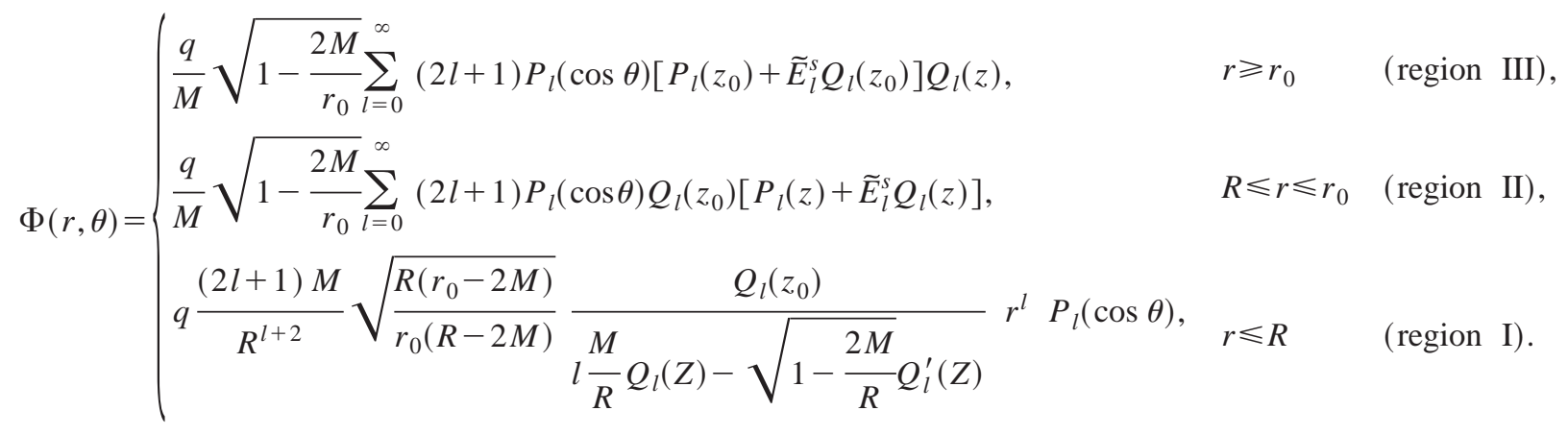

Here, $z \equiv r / M-1, z_{0} \equiv r_{0} / M-1, Z \equiv R / M-1$, and we have used the Wronskian $W\left[P_{n}(z), Q_{n}(z)\right]=-1 /\left(z^{2}-1\right)$ to simplify the expression. We are interested in the field in the neighborhood of the particle. As $r_{0}>R$, the field in this neighborhood can be written as 


$$
\Phi(r>R, \theta)=\frac{q}{M} \sqrt{1-\frac{2 M}{r_{0}}} \sum_{l=0}^{\infty}(2 l+1) P_{l}(\cos \theta)\left[P_{l}\left(z_{0}\right) Q_{l}(z) \Theta\left(r-r_{0}\right)+P_{l}(z) Q_{l}\left(z_{0}\right) \Theta\left(r_{0}-r\right)+\widetilde{E}_{l}^{s} Q_{l}\left(z_{0}\right) Q_{l}(z)\right],
$$

where $\widetilde{E}_{l}^{s}$ is given by

$$
\widetilde{E}_{l}^{s}=\frac{l \frac{M}{R} P_{l}(Z)-\sqrt{1-\frac{2 M}{R}} P_{l}^{\prime}(Z)}{\sqrt{1-\frac{2 M}{R}} Q_{l}^{\prime}(Z)-\frac{l M}{R} Q_{l}(Z)},
$$

which can be shown to be positive for all $l$ and $M / R<1 / 2$.

To find the self-force, we next calculate the force according to $f_{\mu}=q \nabla_{\mu} \Phi$, and evaluate this expression at the position of the particle, i.e., at $r=r_{0}$. Clearly, only the radial component of the force $f_{\mu}$ is non-zero. The (bare) radial force is then given by

$$
\begin{aligned}
f_{r}^{\mathrm{bare}}= & \frac{q^{2}}{M^{2}} \sqrt{1-\frac{2 M}{r_{0}}} \sum_{l=0}^{\infty}(2 l+1)\left\{\frac { 1 } { 2 } \left[P_{l}\left(z_{0}\right) Q_{l}^{\prime}\left(z_{0}\right)\right.\right. \\
& \left.\left.+P_{l}^{\prime}\left(z_{0}\right) Q_{l}\left(z_{0}\right)\right]+\widetilde{E}_{l}^{s} Q_{l}\left(z_{0}\right) Q_{l}^{\prime}\left(z_{0}\right)\right\} .
\end{aligned}
$$

The series, when summed naively, diverges.

\section{Regularization of the bare force}

To regularize the bare force and extract the finite, physical self-force, we use MSRP. Again, only the radial self-force is not trivial. The regularization function $h_{r}=a_{r} l+b_{r}+c_{r} l^{-1}$ can be found from a local analysis near the particle, where spacetime is locally Schwarzschild. In Schwarzschild spacetime the values of $a_{r}, b_{r}, c_{r}$, and $d_{r}$ are completely known for a static scalar particle, and are given by $a_{r}=0=c_{r}$ (we again use here the "averaged" value for $\left.a_{r}\right)$, and $b_{r}=$ $-\left[q^{2} /\left(2 r_{0}^{2}\right)\right]\left[\left(1-M / r_{0}\right) /\left(1-2 M / r_{0}\right)\right]$. In addition, it is also known that $d_{r}=0$. In Ref. [4] it was shown that when MSRP is applied to the term proportional to the square brackets in Eq. (40), the sum over all modes vanishes. That is, when $f_{r}^{\text {bare }}$ is written as a sum over modes, i.e., $f_{r}^{\text {bare }}$ $\equiv \Sigma_{l=0}^{\infty} f_{r}^{\text {bare } l}$, the regularized self-force can be written as $f_{r}=\sum_{l=0}^{\infty}\left(f_{r}^{\text {bare } l}-b_{r}\right)$. Because this series converges, it can be split into two sums, the first of which vanishes [4], such that the regularized, physical self-force in an orthonormal basis is given by

$f_{\hat{r}}=\left(\frac{q}{M}\right)^{2}\left(1-\frac{2 M}{r_{0}}\right) \sum_{l=0}^{\infty}(2 l+1) \widetilde{E}_{l}^{s} Q_{l}\left(\frac{r_{0}}{M}-1\right) Q_{l}^{\prime}\left(\frac{r_{0}}{M}-1\right)$

This is a repulsive force, as it always directs away from the center of the coordinates (and also away from the shell). This result (41) corrects the zero self-force result (for the case where the source of the gravitational field is a black hole $[1,3,4])$ for the finite size of the shell.

\section{Properties of the regularized force}

For small $M / R$, the leading order of Eq. (41) is given by

$$
f_{\hat{r}}=\left(\frac{q}{r_{0}}\right)^{2}\left(\frac{M}{r_{0}}\right)^{2} \sum_{l=1}^{\infty} \frac{l(l+1)}{2(2 l-1)(2 l+1)}\left(\frac{R}{r_{0}}\right)^{2 l-1}
$$

which can be written as

$$
f_{\hat{r}}=q^{2} \frac{G^{2}}{c^{4}} \frac{M^{2}}{r_{0}^{4}}\left[\frac{1+3 y_{0}^{2}}{32 y_{0}^{2}} \ln \left(\frac{1+y_{0}}{1-y_{0}}\right)-\frac{1-3 y_{0}^{2}}{16 y_{0}\left(1-y_{0}^{2}\right)}\right] \text {, }
$$

where $y_{0} \equiv R / r_{0}$. Here, we re-introduced Newton's constant $G$ and the speed of light $c$. As the charge approaches the shell $\left(y_{0} \rightarrow 1^{-}\right)$,

$$
\begin{aligned}
f_{\hat{r}}= & q^{2} \frac{G^{2}}{c^{4}} \frac{M^{2}}{r_{0}^{4}}\left\{\frac{1}{16\left(1-y_{0}\right)}-\frac{1}{8} \ln \left(1-y_{0}\right)-\frac{3}{32}+\frac{\ln 2}{8}\right. \\
& \left.+O\left[\left(1-y_{0}\right) \ln \left(1-y_{0}\right)\right]\right\} .
\end{aligned}
$$

The divergence which we find in the coincidence limit is again due to the simplified model of the pointlike charge (see discussion above). Note, that the result for the self-force in the limit where the particle approaches the shell from the outside (44) is similar to the result for the self-force in the limit where the particle approaches the shell from the inside (25). In fact, the leading order terms (in the inverse of the distance from the shell) of the two cases are identical (they only have opposite signs, because the self-force in both cases repels the particle from the shell). However, the next terms (which are proportional to the logarithm of the distance from the shell) are no longer identical (the relative signs are different) because of the different direction of the shell's curvature with regard to the particle's position.

Figure 3 shows the self-force $f_{\hat{r}}$ as a function of $R / M$ computed by the full expression (41) (solid line) and the small $M / R$ formula (43) (dashed line). The charge is placed at $r_{0} / R=2$. The self-force increases as $R / M$ decreases, reaches a maximum at $R / M \approx 2.3$ and drops to zero when $R / M \rightarrow 2$. It is easy to show analytically from Eqs. (39) and (41) that the self-force vanishes in the limit $R / M \rightarrow 2$, which coincides with the case when the shell is replaced by a Schwarzschild black hole. Of course, in that limit the shell can no longer be static, and must implode instead. The spacetime then is that of a Schwarzschild black hole with appropriate boundary conditions at the event horizon, which are unaffected by the implosion of the shell inside the black hole. That is the reason why the static shell gives the right result (of a zero self-force) in the limit of coincidence of its radius with the Schwarzschild radius.

Finally, Fig. 6(a) displays the magnitude of the self-force $\left|f_{\hat{r}}\right|$ as a function of $r_{0} / R$ for $R=2.5 M$. We can see that the 


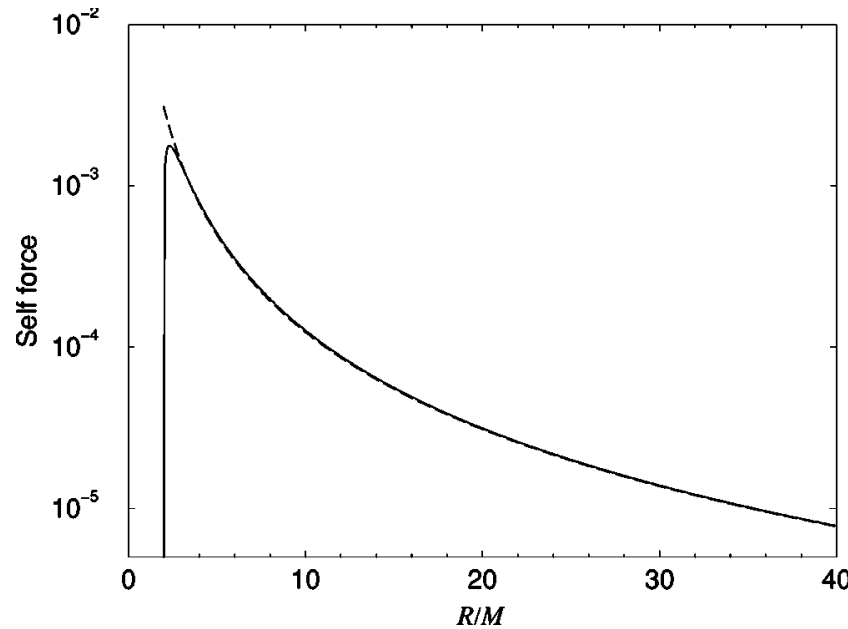

FIG. 3. Same as Fig. 2 but the scalar charge is placed outside the shell at $r_{0} / R=2$. Solid line is computed by the full expression Eq. (41); dashed line is computed by the small $M / R$ formula (43). The self-force vanishes in the limit $R / M \rightarrow 2$.

small $M / R$ expressions (dashed line) are no longer accurate in this case. The self force decreases as the charge moves away from the shell, but diverges at $r_{0}=R$ (see the above discussion).

\section{Alternative regularization procedure}

Again, the regularization prescription can be performed also by applying directly Quinn's comparison axiom. This time, we choose the spacetime of a globally-Schwarzschild spacetime (a Schwarzschild black hole) as the "tilde" spacetime. That is, we write the total scalar field as

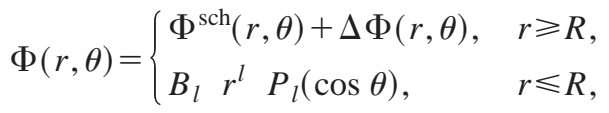

where

$$
\begin{aligned}
B_{l}= & q \frac{(2 l+1) M}{R^{l+2}}\left(1-\frac{2 M}{r_{0}}\right)^{1 / 2}\left(1-\frac{2 M}{R}\right)^{-1 / 2} \\
& \times \frac{Q_{l}\left(\frac{r_{0}}{M}-1\right)}{l \frac{M}{R} Q_{l}\left(\frac{R}{M}-1\right)-\sqrt{1-\frac{2 M}{R}} Q_{l}^{\prime}\left(\frac{R}{M}-1\right)},
\end{aligned}
$$

and

$$
\begin{aligned}
\Phi^{\operatorname{sch}}(r, \theta)= & \frac{q}{M} \sqrt{1-\frac{2 M}{r_{0}}} \sum_{l=0}^{\infty}(2 l+1) P_{l}(\cos \theta) \\
& \times\left[P_{l}\left(\frac{r_{0}}{M}-1\right) Q_{l}\left(\frac{r}{M}-1\right) \Theta\left(r-r_{0}\right)\right. \\
& \left.+P_{l}\left(\frac{r}{M}-1\right) Q_{l}\left(\frac{r_{0}}{M}-1\right) \Theta\left(r_{0}-r\right)\right]
\end{aligned}
$$

is the scalar field (which we choose to be $\tilde{\phi}$ ) for the case where the shell is replaced by a Schwarzschild black hole of the same mass [4], and

$$
\begin{aligned}
\Delta \Phi(r, \theta)= & \frac{q}{M} \sqrt{1-\frac{2 M}{r_{0}}} \sum_{l=0}^{\infty}(2 l+1) \widetilde{E}_{l}^{s} Q_{l}\left(\frac{r_{0}}{M}-1\right) \\
& \times Q_{l}\left(\frac{r}{M}-1\right) P_{l}(\cos \theta),
\end{aligned}
$$

is the correction due to the finiteness of the shell's radius. Applying the comparison axiom, the self-force is given by

$f_{r}=q(\Delta \Phi)_{, r}$

$f_{\hat{r}}=\left(\frac{q}{M}\right)^{2}\left(1-\frac{2 M}{r_{0}}\right) \sum_{l=0}^{\infty}(2 l+1) \widetilde{E}_{l}^{s} Q_{l}\left(\frac{r_{0}}{M}-1\right) Q_{l}^{\prime}\left(\frac{r_{0}}{M}-1\right)$,

which is identical to Eq. (41). [This can also be stated, alternatively, in the following way: Because the self force is zero when the source of the gravitational field is point-like, the self-force is given only by $f_{r}=q(\Delta \Phi){ }_{, r}$.]

\section{ELECTRIC CHARGE}

The second type of charge we study is an electric charge $q$, which again, following the preceding section, is at rest inside or outside a spherical shell of mass $M$. In this case, we look for the solution of the Maxwell equation

$$
\nabla_{\nu} F^{\mu \nu}=4 \pi j^{\mu}
$$

where $F_{\mu \nu}$ is the Maxwell field-strength tensor, which is antisymmetric and related to the 4-vector potential $A_{\mu}$ by $F_{\mu \nu}=A_{\nu, \mu}-A_{\mu, \nu}$. Here, $j^{\mu}=\rho u^{\mu}$ is the four-current density, $\rho$ is the charge density, and $u^{\mu}$ is the 4-velocity of the charge. Since the charge is static, the spatial components of $j^{\mu}$ vanish. From the spatial components of Eq. (50), one can easily show that it is possible to choose a gauge so that all spatial components of $A_{\mu}$ vanish. The equation for $A_{t}$ is given by the temporal component of Eq. (50):

$$
\frac{1}{\sqrt{-g}}\left(\sqrt{-g} g^{\nu \alpha} g^{t t} A_{t, \alpha}\right)_{, \nu}=-4 \pi j^{t}
$$

with $j^{t}$ given by

$$
j^{t}=q \int_{-\infty}^{\infty} u^{t} \frac{\delta^{4}\left[x^{\mu}-z^{\mu}(\tau)\right]}{\sqrt{-g}} d \tau
$$

\section{A. Electric charge inside the shell}

\section{Derivation of the bare force}

For the case where the electric charge is inside the shell, $r_{0}<R$. This case was studied by Unruh in Ref. [12]. The decomposition of $A_{t}$ and $j^{t}$ into modes yields 


$$
\begin{aligned}
A_{t}(r, \theta)= & \sum_{l=0}^{\infty} \phi^{l}(r) P_{l}(\cos \theta) \\
j^{t}(r, \theta)= & \frac{q}{4 \pi} \frac{\delta\left(r-r_{0}\right)}{r_{0}^{2}}\left(1-\frac{2 M}{R}\right)^{-1 / 2} \\
& \times \sum_{l=0}^{\infty}(2 l+1) P_{l}(\cos \theta) .
\end{aligned}
$$

Substituting these decompositions into Eq. (51) we find that the latter becomes

$$
\begin{array}{r}
\phi_{, r r}^{l}+\frac{2}{r} \phi_{, r}^{l}-\left(1-\frac{2 M}{r}\right)^{-1} \frac{l(l+1)}{r^{2}} \phi^{l}=0, \quad r>R, \\
\phi_{, r r}^{l}+\frac{2}{r} \phi_{, r}^{l}-\frac{l(l+1)}{r^{2}} \phi^{l}= \\
\quad \frac{(2 l+1)}{r_{0}^{2}} \sqrt{1-\frac{2 M}{R}} \\
\times \delta\left(r-r_{0}\right), \quad r<R .
\end{array}
$$

$$
\lim _{\epsilon \rightarrow 0^{+}}\left[\phi_{, r}^{l}\left(r_{0}+\epsilon\right)-\phi_{, r}^{l}\left(r_{0}-\epsilon\right)\right]=q \frac{2 l+1}{r_{0}^{2}} \sqrt{1-\frac{2 M}{R}},
$$

which comes from integrating Eq. (55) across $r=r_{0}$. The time component of the 4-vector potential $A_{\mu}$, the only nonvanishing component in our gauge, in a normalized basis is then

$$
A^{t}(r, \theta)=\left\{\begin{array}{lll}
\sum_{l=0}^{\infty} G_{l}(r-2 M) Q_{l}^{\prime}\left(\frac{r}{M}-1\right) P_{l}(\cos \theta), & r \geqslant R & \text { (region III), } \\
\sum_{l=0}^{\infty} q \frac{r_{0}^{l}}{r^{l+1}} P_{l}(\cos \theta)+\sum_{l=0}^{\infty} \frac{q}{R}\left(\frac{r_{0}}{R}\right)^{l}\left(\frac{r}{R}\right)^{l} E_{l}^{e} P_{l}(\cos \theta), & r_{0} \leqslant r \leqslant R & \text { (region II), } \\
\sum_{l=0}^{\infty} q \frac{r^{l}}{r_{0}^{l+1}} P_{l}(\cos \theta)+\sum_{l=0}^{\infty} \frac{q}{R}\left(\frac{r_{0}}{R}\right)^{l}\left(\frac{r}{R}\right)^{l} E_{l}^{e} P_{l}(\cos \theta), & r \leqslant r_{0} & \text { (region I), }
\end{array}\right.
$$

where

$$
G_{l}=-q \frac{2 l+1}{\sqrt{1-\frac{2 M}{r}}} \frac{M r_{0}^{l}}{R^{l+3}}\left[\left(1-\frac{2 M}{R}\right) Q_{l}^{\prime \prime}\left(\frac{R}{M}-1\right)-\frac{M}{R}\left(l \sqrt{1-\frac{2 M}{R}}-1\right) Q_{l}^{\prime}\left(\frac{R}{M}-1\right)\right]^{-1}
$$

and

$$
E_{l}^{e}=\frac{\frac{M}{R}\left[(l+1) \sqrt{\left.1-\frac{2 M}{R}+1\right]} Q_{l}^{\prime}\left(\frac{R}{M}-1\right)+\left(1-\frac{2 M}{R}\right) Q_{l}^{\prime \prime}\left(\frac{R}{M}-1\right)\right.}{\frac{M}{R}\left(l \sqrt{\left.1-\frac{2 M}{R}-1\right)} Q_{l}^{\prime}\left(\frac{R}{M}-1\right)-\left(1-\frac{2 M}{R}\right) Q_{l}^{\prime \prime}\left(\frac{R}{M}-1\right)\right.} .
$$

In the neighborhood of the charge the potential then is given by

$$
A^{\hat{t}}(r, \theta)=\sum_{l=0}^{\infty} q\left[\frac{r^{l}}{r_{0}^{l+1}} \Theta\left(r_{0}-r\right)+\frac{r_{0}^{l}}{r^{l+1}} \Theta\left(r-r_{0}\right)\right] P_{l}(\cos \theta)+\sum_{l=0}^{\infty} \frac{q}{R}\left(\frac{r_{0}}{R}\right)^{l}\left(\frac{r}{R}\right)^{l} E_{l}^{e} P_{l}(\cos \theta)
$$


The self-force is computed by calculating the Lorentz force arising from this potential, and evaluating it at the position of the charge. We thus find the bare force to be

$$
f_{r}^{\text {bare }}=\sum_{l=0}^{\infty}\left[-\frac{q^{2}}{2 r_{0}^{2}}-\left(\frac{q}{R}\right)^{2} l E_{l}^{e}\left(\frac{r_{0}}{R}\right)^{2 l-1}\right] .
$$

This (bare) force diverges when the series is naively summed.

\section{Regularization of the bare force}

Next, we regularize this force using MSRP. Although MSRP has been developed only for scalar charges, it was shown in Refs. $[4,22]$ that it is also applicable for electric charges, at least in simple cases. In particular, for an electric charge at rest in a locally-Minkowski spacetime, the values of the regularization functions are known, and are given by $a_{r}=0=c_{r}, b_{r}=-q^{2} /\left(2 r_{0}^{2}\right)$, and $d_{r}=0$. Consequently, the regularized, physical self-force is given by

$$
f_{r}=-\left(\frac{q}{R}\right)^{2} \sum_{l=0}^{\infty} l E_{l}^{e}\left(\frac{r_{0}}{R}\right)^{2 l-1} .
$$

\section{Properties of the regularized force}

When $E_{l}^{e}$ is expanded in $M / R$ we find

$$
E_{l}^{e}=\frac{1}{2 l+1}\left(\frac{M}{R}\right)+O\left(\frac{M}{R}\right)^{2},
$$

such that

$$
f_{r}=-\left(\frac{q}{R}\right)^{2} \frac{M}{R}\left[\sum_{l=1}^{\infty} \frac{l}{2 l+1}\left(\frac{r_{0}}{R}\right)^{2 l-1}+O\left(\frac{M}{R}\right)\right] .
$$

To the leading order in $M / R$ this result can be written as

$$
f_{r}=-q^{2} \frac{G}{c^{2}} \frac{M}{R^{3}} \frac{1}{2 x_{0}}\left[\frac{1}{1-x_{0}^{2}}-\frac{1}{2 x_{0}} \ln \left(\frac{1+x_{0}}{1-x_{0}}\right)\right],
$$

where $x_{0}=r_{0} / R$. This force is directed toward the center, or away from the sphere, and in this sense it is a repelling force. In Eq. (65) we re-introduced Newton's constant $G$ and the speed of light $c$. This force is a 1st post-Newtonian effect.

When the charge is close to the center, the self-force is proportional to $r$, and the charge will oscillate with frequency

$$
\begin{aligned}
\omega & =\sqrt{\frac{1}{3} q^{2} \frac{G}{c^{2}} \frac{M}{m R^{4}}} \\
& =3.9 \times 10^{-5} \frac{\mathrm{rad}}{\mathrm{s}}\left(\frac{q}{e}\right)\left(\frac{M}{M_{\odot}}\right)^{1 / 2}\left(\frac{m}{m_{e}}\right)^{-1 / 2}\left(\frac{R}{3 \mathrm{~km}}\right)^{-2},
\end{aligned}
$$

where $m$ is the charge's mass, $e$ is electron's charge, $m_{e}$ is the electron's mass, and $M_{\odot}$ is solar mass. This frequency is small, and is unlikely of being detected. Next we consider the force to the leading orders in $1-x_{0}$, and find that

$$
\begin{aligned}
f_{r}= & -q^{2} \frac{G}{c^{2}} \frac{M}{R^{3}}\left\{\frac{1}{4\left(1-x_{0}\right)}+\frac{1}{4} \ln \left(1-x_{0}\right)+\frac{3}{8}-\frac{\ln 2}{4}\right. \\
& \left.+O\left[\left(1-x_{0}\right) \ln \left(1-x_{0}\right)\right]\right\} .
\end{aligned}
$$

Again, this force diverges in the coincidence limit $\left(x_{0}\right.$ $\rightarrow 1^{-}$), but this divergence only signifies the breakdown of our model at this limit.

Figure 4 shows the self force $f_{r}$ as a function of $R / M$ computed by full expression (62) (solid line) and the small $M / R$ expression (65) (dashed line). The result is very similar to the scalar charge case (cf. Fig. 2). It can be shown that

$$
f_{r} \rightarrow-\frac{q^{2}}{R^{2}} \frac{G}{c^{2}} \frac{2 x_{0}-x_{0}^{3}}{\left(1-x_{0}^{2}\right)^{2}}
$$

as $R / M \rightarrow 2$.

\section{Alternative regularization procedure}

Similarly to the direct application of the comparison axiom in the scalar case, we can apply the electric-field comparison axiom [16] here analogously. The electric field comparison axiom is nearly identical to the scalar-field comparison axiom. In fact, all that we need to change in its definition is to replace the scalar fields $\phi$ and $\widetilde{\phi}$ with the Maxwell fields $F_{\mu \nu}$ and $\widetilde{F}_{\mu \nu}$, respectively, and replace Eq. (27) with

$$
f_{\mu}-\widetilde{f}_{\mu}=\lim _{x \rightarrow 0} q\left\langle F_{\mu \nu}-\widetilde{F}_{\mu \nu}\right\rangle_{x} u^{\nu} .
$$

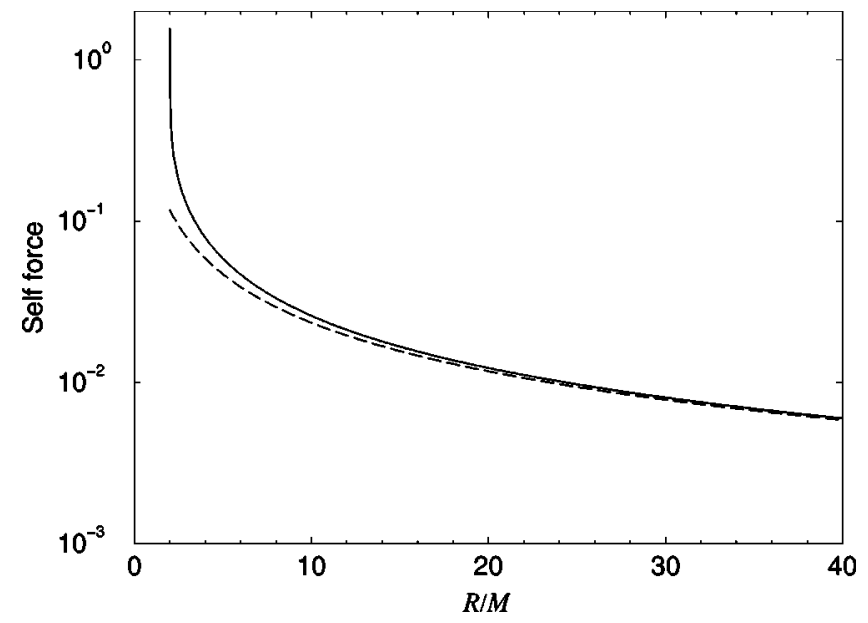

FIG. 4. The magnitude of the self-force $\left|f_{\hat{r}}\right|$, in units of $q^{2} G /\left(R^{2} c^{2}\right)$, experienced by an electric charge as a function of $R / M$, computed by the full expression Eq. (62) (solid line) and the small $M / R$ formula (65) (dashed line). The charge is placed inside the shell at $r_{0} / R=1 / 2$, and the self-force approaches the limit $(14 / 9) q^{2} G /\left(R^{2} c^{2}\right)$ predicted by Eq. (69) when $R / M \rightarrow 2$. 
In order to apply the comparison axiom we write the potential in the form

$$
\begin{aligned}
& A^{\hat{t}(r, \theta)} \\
& \quad= \begin{cases}\sum_{l=0}^{\infty} G_{l}(r-2 M) Q_{l}^{\prime}\left(\frac{r}{M}-1\right) P_{l}(\cos \theta), & r \geqslant R, \\
\Phi_{c}(r, \theta)+\Delta \Phi_{e}(r, \theta), & r \leqslant R,\end{cases}
\end{aligned}
$$

where $\Phi_{c}$ is the Coulomb field (29), which is the potential which is used to construct $\widetilde{F}_{\mu \nu}$, and

$$
\Delta \Phi_{e}(r, \theta)=\sum_{l=0}^{\infty} \frac{q}{R}\left(\frac{r_{0}}{R}\right)^{l}\left(\frac{r}{R}\right)^{l} E_{l}^{e} P_{l}(\cos \theta)
$$

is the correction term due to the finite size of the Minkowski patch of spacetime. Applying the comparison axiom, we find that the self-force is given by

$$
f_{r}=-\left(\frac{q}{R}\right)^{2} \sum_{l=1}^{\infty} l E_{l}^{e}\left(\frac{r_{0}}{R}\right)^{2 l-1},
$$

which is identical to Eq. (62).

\section{B. Electric charge outside the shell}

\section{Derivation of the bare force}

Next, we shall study the case where the electric charge $q$ is outside the shell, i.e., the case where $r_{0}>R$. The timecomponent of the 4-current density is now decomposed as

$$
j^{t}=\frac{q}{4 \pi} \frac{\delta\left(r-r_{0}\right)}{r_{0}^{2}} \sum_{l=0}^{\infty}(2 l+1) P_{l}(\cos \theta) .
$$

With $A_{t}$ decomposed as in Eq. (53), the Maxwell equation (50) becomes

$$
\begin{gathered}
\phi_{, r r}^{l}+\frac{2}{r} \phi_{, r}^{l}-\left(1-\frac{2 M}{r}\right)^{-1} \frac{l(l+1)}{r^{2}} \phi^{l} \\
=q(2 l+1) \frac{\delta\left(r-r_{0}\right)}{r^{2}}, \quad r>R,
\end{gathered}
$$

$$
\phi_{, r r}^{l}+\frac{2}{r} \phi_{, r}^{l}-\frac{l(l+1)}{r^{2}} \phi^{l}=0, \quad r<R .
$$

Hence, $\phi^{l}$ can be written as

$$
\phi^{l}(r)=\left\{\begin{array}{lll}
A_{l}(r-2 M) Q_{l}^{\prime}\left(\frac{r}{M}-1\right), & r \geqslant r_{0} & \text { (region III), } \\
C_{l}(r-2 M) P_{l}^{\prime}\left(\frac{r}{M}-1\right)+D_{l}(r-2 M) Q_{l}^{\prime}\left(\frac{r}{M}-1\right), & R \leqslant r \leqslant r_{0} & \text { (region II), } \\
B_{l} r^{l}, & r \leqslant R & \text { (region I), }
\end{array}\right.
$$

when $l \neq 0$. When $l=0$ the solution has the form

$$
\phi^{0}(r)=\left\{\begin{array}{lll}
\frac{A_{0}}{r}, & r \geqslant r_{0} & (\text { region III) }, \\
C_{0}+\frac{D_{0}}{r}, & R \leqslant r \leqslant r_{0} & (\text { region II) } \\
B_{0}, & r \leqslant R & \text { (region I) } .
\end{array}\right.
$$

$A_{l}, B_{l}, C_{l}$ and $D_{l}$ are constants to be determined by the matching conditions, which are the same as the case when the electric charge is inside the shell, except that Eq. (56) is replaced by

$$
\lim _{\epsilon \rightarrow 0^{+}}\left[\phi_{, r}^{l}\left(r_{0}+\epsilon\right)-\phi_{, r}^{l}\left(r_{0}-\epsilon\right)\right]=q \frac{2 l+1}{r_{0}^{2}} .
$$

Straightforward calculations yield 


$$
A_{t}(r, \theta)= \begin{cases}-\frac{q}{r}+\sum_{l=1}^{\infty} \frac{q}{M^{3}} \frac{2 l+1}{l(l+1)}(r-2 M)\left(r_{0}-2 M\right) P_{l}(\cos \theta)\left[P_{l}^{\prime}\left(z_{0}\right) Q_{l}^{\prime}(z)+\widetilde{E}_{l}^{e} Q_{l}^{\prime}\left(z_{0}\right) Q_{l}^{\prime}(z)\right], & r \geqslant r_{0}, \\ -\frac{q}{r_{0}}+\sum_{l=1}^{\infty} \frac{q}{M^{3}} \frac{2 l+1}{l(l+1)}(r-2 M)\left(r_{0}-2 M\right) P_{l}(\cos \theta)\left[P_{l}^{\prime}(z) Q_{l}^{\prime}\left(z_{0}\right)+\widetilde{E}_{l}^{e} Q_{l}^{\prime}\left(z_{0}\right) Q_{l}^{\prime}(z)\right], & R \leqslant r \leqslant r_{0}, \\ -\frac{q}{r_{0}}+\sum_{l=1}^{\infty} q \frac{M r_{0}}{R^{l+3}} \frac{(2 l+1)\left(1-\frac{2 M}{r_{0}}\right) Q_{l}^{\prime}\left(z_{0}\right)}{\left(1-\frac{2 M}{R}\right) Q_{l}^{\prime \prime}(Z)-\frac{M}{R}\left(l \sqrt{1-\frac{2 M}{R}}-1\right) Q_{l}^{\prime}(Z)} P_{l}(\cos \theta), & r \leqslant R .\end{cases}
$$

Here,

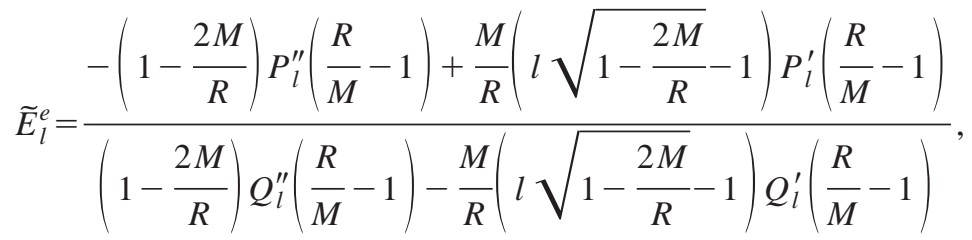

and we have used the Wronskian $W\left[P_{n}^{\prime}(z), Q_{n}^{\prime}(z)\right]=n(n+1) /\left(z^{2}-1\right)^{2}$ to simplify the expressions. In the neighborhood of the charge the potential can thus be written as

$$
\begin{aligned}
A_{t}= & -\frac{q}{r} \Theta\left(r-r_{0}\right)-\frac{q}{r_{0}} \Theta\left(r_{0}-r\right)+\sum_{l=1}^{\infty} \frac{q}{M^{3}} \frac{2 l+1}{l(l+1)}(r-2 M)\left(r_{0}-2 M\right) P_{l}(\cos \theta)\left[P_{l}^{\prime}(z) Q_{l}^{\prime}\left(z_{0}\right) \Theta\left(r_{0}-r\right)\right. \\
& \left.+P_{l}^{\prime}\left(z_{0}\right) Q_{l}^{\prime}(z) \Theta\left(r-r_{0}\right)+\widetilde{E}_{l}^{e} Q_{l}^{\prime}\left(z_{0}\right) Q_{l}^{\prime}(z)\right] .
\end{aligned}
$$

The (bare) force is given formally by

$$
f_{r}^{\mathrm{bare}}=q F_{r \mu} u^{\mu}=q\left(1-\frac{2 M}{r_{0}}\right)^{-1 / 2} A_{t, r},
$$

which of course diverges.

\section{Regularization of the bare force}

The application of MSRP to this case is not straightforward, because the analytical value of $b_{r}$ is as yet unknown. However, it was shown in Ref. [4] that it can be computed numerically, by studying the large- $l$ behavior of the modes of the bare force. It was also found numerically in Ref. [4] that for an electric charge at rest in Schwarzschild spacetime the values of the other regularization functions are given by $a_{r}=0=c_{r}$ and that $d_{r}=0$. When MSRP is applied, the regularized, physical self-force in an orthonormal frame is found to be given by

$$
\begin{aligned}
f_{\hat{r}}= & q^{2} \frac{M}{r_{0}^{3}}+\frac{q^{2} r_{0}}{M^{3}}\left(1-\frac{2 M}{r_{0}}\right) \sum_{l=1}^{\infty} \frac{2 l+1}{l(l+1)} \widetilde{E}_{l}^{e}\left[Q_{l}^{\prime}\left(\frac{r_{0}}{M}-1\right)\right]^{2} \\
& +\frac{q^{2} r_{0}^{2}}{M^{4}}\left(1-\frac{2 M}{r_{0}}\right)^{2} \sum_{l=1}^{\infty} \frac{2 l+1}{l(l+1)} \widetilde{E}_{l}^{e} Q_{l}^{\prime}\left(\frac{r_{0}}{M}-1\right) \\
& \times Q_{l}^{\prime \prime}\left(\frac{r_{0}}{M}-1\right)
\end{aligned}
$$

\section{Properties of the regularized force}

To leading order in $M / R$, the self-force is reduced to the following simple expression:

$$
f_{\hat{r}}=\frac{q^{2} M}{r_{0}^{3}} \sum_{l=0}^{\infty} \frac{l+1}{2 l+1}\left(\frac{R}{r_{0}}\right)^{2 l},
$$

which can be written as

$$
f_{\hat{r}}=q^{2} \frac{G}{c^{2}} \frac{M}{2 r_{0}^{3}}\left[\frac{1}{1-y_{0}^{2}}+\frac{1}{2 y_{0}} \ln \left(\frac{1+y_{0}}{1-y_{0}}\right)\right],
$$

where $y_{0}=R / r_{0}$. In the last equation we re-introduced Newton's constant $G$ and the speed of light $c$. The effect of the finite size of the shell is to increase the self-force repulsion which was found first by Smith and Will for the case where the source of the gravitational field is a (Schwarzschild) black hole [2]. To leading orders in $1-y_{0}$ we find that the force is given by

$$
\begin{aligned}
f_{\hat{r}}= & q^{2} \frac{G}{c^{2}} \frac{M}{r_{0}^{3}}\left\{\frac{1}{4\left(1-y_{0}\right)}-\frac{1}{4} \ln \left(1-y_{0}\right)+\frac{1}{8}+\frac{\ln 2}{4}\right. \\
& \left.+O\left[\left(1-y_{0}\right) \ln \left(1-y_{0}\right)\right]\right\} .
\end{aligned}
$$



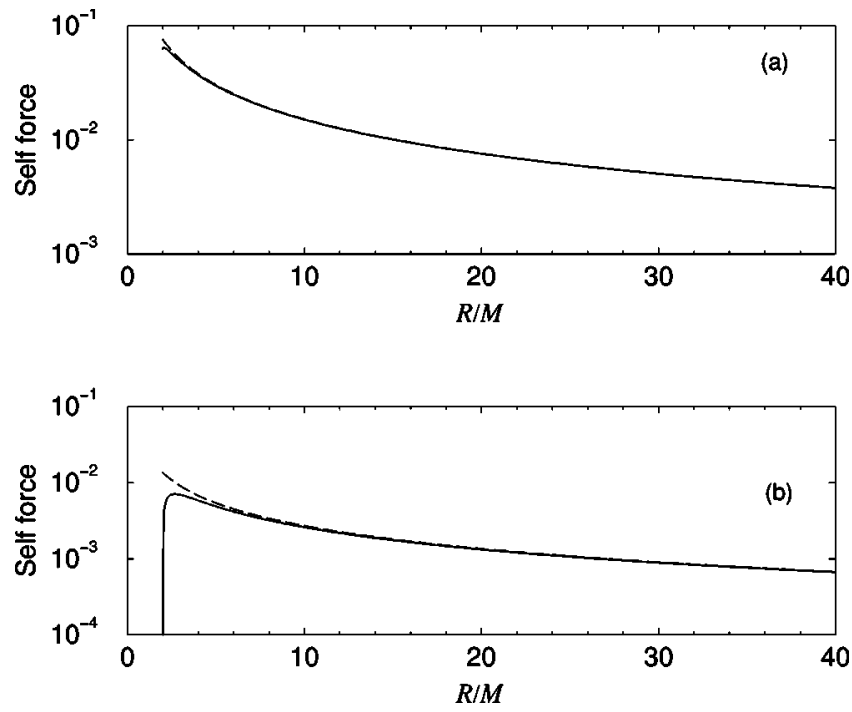

FIG. 5. Same as Fig. 4 but the electric charge is placed outside the shell at $r_{0} / R=2$. The upper panel (a) shows the total self-force. The lower panel (b) shows the correction to the self-force due to the finite size of the Minkowski patch [i.e. $\Delta f_{\hat{r}}$ in Eq. (92)]. Solid lines are calculated by the full expression Eq. (83); dashed lines are calculated based on the small $M / R$ formula (85). The self-force approaches the Smith-Will result when $R / M \rightarrow 2$.

This force diverges in the coincidence limit $\left(y_{0} \rightarrow 1^{-}\right)$, again, due to the breakdown of the mathematical model.

Figure 5 shows (a) the total self-force and (b) the correction of the self-force due to the finite Minkowski patch [i.e. $\Delta f_{\hat{r}}$ in Eq. (92)] in unit of $q^{2} G /\left(c^{2} R^{2}\right)$ as a function of $R / M$ based on the full expression (83) and the small $M / R$ formula (85). The charge is placed at $r_{0} / R=2$. We can see that the situation is very similar to the scalar case (cf. Fig. 3). The self-force reduces to the Will-Smith result in the limit $R / M$ $\rightarrow 2$, as expected.

Finally, Fig. 6(b) shows the magnitude of the self-force in unit of $q^{2} G /\left(c^{2} R^{2}\right)$ as a function of $r_{0} / R$ for $R=2.5 M$. We can see that the small $M / R$ expressions (dashed line) deviates significantly from the full expression (solid line) when the charge is inside the shell, but is quite accurate when it is outside the shell. The reason is that the self-force is dominated by the Smith-Will force, which are present in both the full and the small $M / R$ expressions, when the charge is outside the shell. The self-force decreases as the charge moves away from the shell, but diverges at $r_{0}=R$ (see the above discussion).

\section{Alternative regularization procedure}

Again, we can apply the comparison axiom directly. We thus write the potential as

$$
A_{t}= \begin{cases}A_{t}^{\mathrm{sch}}+\Delta A_{t}, & r \geqslant R, \\ B_{l} r^{l} P_{l}(\cos \theta), & r \leqslant R,\end{cases}
$$
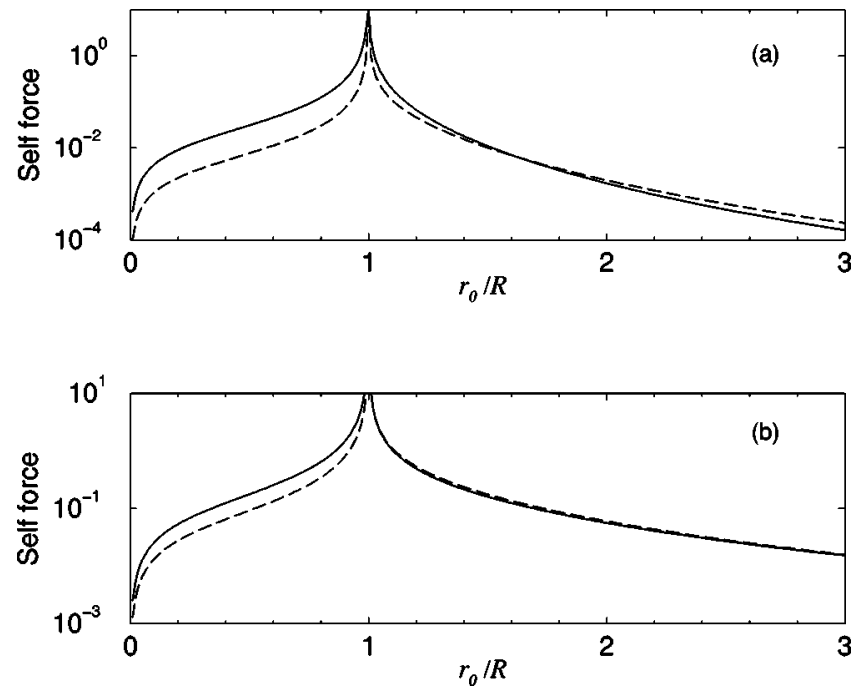

FIG. 6. The magnitude of the self-force $\left|f_{\hat{r}}\right|$ experienced by (a) a scalar charge [in units of $q^{2} G^{2} /\left(c^{4} R^{2}\right)$ ] and (b) an electric charge [in units of $q^{2} G /\left(c^{2} R^{2}\right)$ ] as a function of the charge's position $r_{0}$. The radius of the shell is set to $R=2.5 \mathrm{M}$. Solid lines are calculated by corresponding full expressions and dashed lines are calculated by the appropriate expressions for small $M / R$.

where

$$
\begin{aligned}
A_{t}^{\mathrm{sch}}= & -\frac{q}{r} \Theta\left(r-r_{0}\right)-\frac{q}{r_{0}} \Theta\left(r_{0}-r\right) \\
& +\sum_{l=1}^{\infty} \frac{q}{M^{3}} \frac{2 l+1}{l(l+1)}(r-2 M)\left(r_{0}-2 M\right) P_{l}(\cos \theta) \\
& \times\left[P_{l}^{\prime}\left(\frac{r}{M}-1\right) Q_{l}^{\prime}\left(\frac{r_{0}}{M}-1\right) \Theta\left(r_{0}-r\right)\right. \\
& \left.+P_{l}^{\prime}\left(\frac{r_{0}}{M}-1\right) Q_{l}^{\prime}\left(\frac{r}{M}-1\right) \Theta\left(r-r_{0}\right)\right]
\end{aligned}
$$

is the potential if the shell is replaced by a Schwarzschild black hole of the same mass [4] (i.e., a point-like source for the gravitational field), which is the origin for $\widetilde{F}_{\mu \nu}$, and

$$
\begin{aligned}
\Delta A_{t}= & \frac{q}{M^{3}}\left(r_{0}-2 M\right)(r-2 M) \sum_{l=1}^{\infty} \frac{2 l+1}{l(l+1)} \widetilde{E}_{l}^{e} \\
& \times Q_{l}^{\prime}\left(\frac{r_{0}}{M}-1\right) Q_{l}^{\prime}\left(\frac{r}{M}-1\right) P_{l}(\cos \theta)
\end{aligned}
$$

is the correction for the potential because of the finite size of the Minkowski patch of spacetime. Here, 


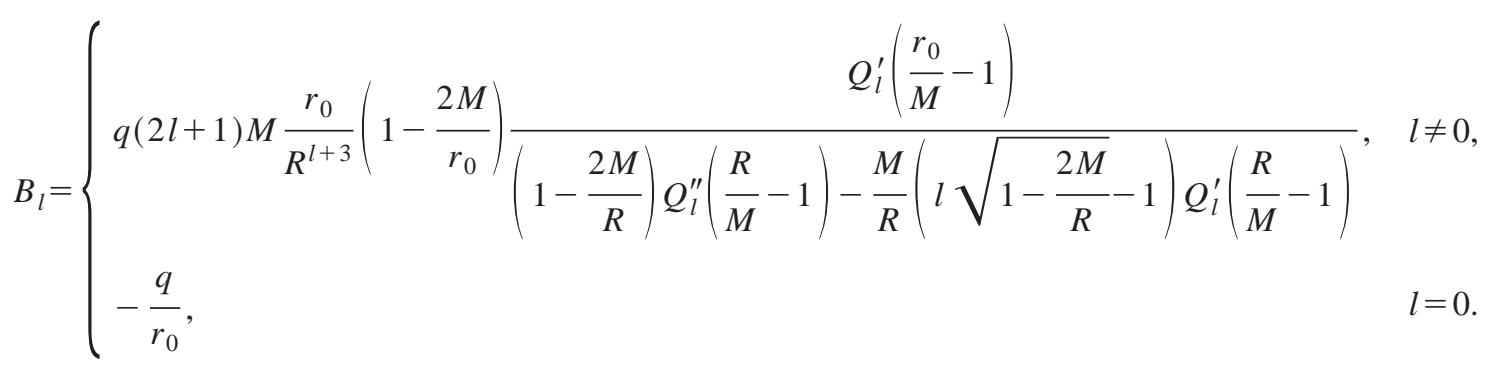

The difference in the self-forces between the actual and the "tilde" spacetimes is then given by

$$
f_{r}-\widetilde{f}_{r}=q\left(F_{r \mu}-\widetilde{F}_{r \mu}\right) u^{\mu}=q\left(1-\frac{2 M}{r_{0}}\right)^{-1 / 2}\left(\Delta A_{t}\right)_{, r} .
$$

This difference then equals

$$
\begin{aligned}
f_{r}-\widetilde{f}_{r} \equiv \Delta f_{r}= & q\left(1-\frac{2 M}{r_{0}}\right)^{-1 / 2} \Delta A_{t, r} \\
f_{\hat{r}}= & \frac{q^{2} r_{0}}{M^{3}}\left(1-\frac{2 M}{r_{0}}\right) \sum_{l=1}^{\infty} \frac{2 l+1}{l(l+1)} \widetilde{E}_{l}^{e}\left[Q_{l}^{\prime}\left(\frac{r_{0}}{M}-1\right)\right]^{2} \\
& +\frac{q^{2} r_{0}^{2}}{M^{4}}\left(1-\frac{2 M}{r_{0}}\right)^{2} \sum_{l=1}^{\infty} \frac{2 l+1}{l(l+1)} \widetilde{E}_{l}^{e} \\
& \times Q_{l}^{\prime}\left(\frac{r_{0}}{M}-1\right) Q_{l}^{\prime \prime}\left(\frac{r_{0}}{M}-1\right) .
\end{aligned}
$$

Recall now that $f_{\hat{r}}=\Delta f_{\hat{r}}+\tilde{f}_{\hat{r}}$, where $\tilde{f}_{\hat{r}}$ is the Smith-Will force given by $\tilde{f}_{\hat{r}}=q^{2}\left(M / r_{0}^{3}\right)$. When combined, we recover the self force $f_{\hat{r}}$, which is identical to Eq. (83).

\section{HEURISTIC VIEWPOINT ON THE ORIGIN OF THE SELF-FORCE}

The self-force experienced by a static electric charge in the presence of the shell can be interpreted as a result of the interaction between the charge's electric field and the shell's gravitational field. In this section, we shall consider the self force in the small $M / R$ limit, i.e. Eqs. (65) and (85), by a heuristic argument. It is the linearization in $M / R$ which allows us to obtain the solution very easily.

Consider a static electric charge $q$ at the origin of the coordinate system. Spacetime is then described by the Reissner-Nordström metric

$$
\begin{aligned}
d s^{2}= & -\left(1-\frac{2 m}{r}+\frac{q^{2}}{r^{2}}\right) d t^{2}+\left(1-\frac{2 m}{r}+\frac{q^{2}}{r^{2}}\right)^{-1} d r^{2} \\
& +r^{2}\left(d \vartheta^{2}+\sin ^{2} \vartheta d \varphi^{2}\right),
\end{aligned}
$$

where $m$ is the mass of the charge. A particle of mass $\mu$ fixed at $r$ will experience a "gravitational force" $f^{\alpha}$ $=-\mu D u^{\alpha} / D \tau$, where $u^{\alpha}$ is the 4-velocity and $D / D \tau$ denotes covariant derivative [compatible with the metric (93)] with respect to the particle's proper time. Because of the symmetry of the setup, the only non-vanishing component of $f^{\alpha}$, in an orthonormal basis, is

$$
f^{r}=-\mu\left(u^{t}\right)^{2} \Gamma_{t t}^{r}\left(1-\frac{2 m}{r}+\frac{q^{2}}{r^{2}}\right)^{-1 / 2}=-\frac{\mu m}{r^{2}}+\frac{\mu q^{2}}{r^{3}}
$$

to leading order in $m$ and $q^{2}$, where $\Gamma_{\beta \gamma}^{\alpha}$ are the connection coefficients. The first term is the usual (attractive) gravitational force, whereas the second term, which is a repulsive force, comes from the stress-energy tensor of the electric field. In what follows we shall ignore the former, as we are interested only in the electric-field interaction and not in the direct gravitational force. Because of the staticity of the problem, the two-body system (charge and massive particle) conserves linear momentum. Consequently, Newton's third law is applicable for this two-body system. (In general, when radiation is present, Newton's third law is inapplicable.) It follows from Newton's third law that the static electric charge also experiences this repulsive force $q^{2} \mu / r^{3}$ apart from the usual attractive gravitational force. This additional repulsive force on the static charge is then interpreted as a result of the interaction between the charge's electric field and the point mass $\mu$, i.e., it is the self-force. Notice that this result is the same as the self force acting on a static electric charge in Schwarzschild spacetime computed by Smith and Will [2] (notice, however, that by this argument we only find the leading order term in $\mu$. The Smith-Will force, however, is an exact result).

Now, suppose the charge is surrounded by a spherical shell of radius $R$ and mass $M$ [Fig. 7(a)]. In the small $M / R$ and test charge limits, the "gravitational force" acting on the shell by the charge's electric field is equal to the sum of the forces acting on each particle on the shell. We express the mass $\mu$ of a small element of the shell by $\mu$ $=M d^{2} x /\left(4 \pi R^{2}\right)$, where $d^{2} x$ is an area element of the shell. From symmetry, it is clear that the total force is aligned on the $z$-axis. We thus project all the contributions to the total force on this axis, such that the vector summation becomes trivial. The resultant force is along the $z$-axis and is given by 


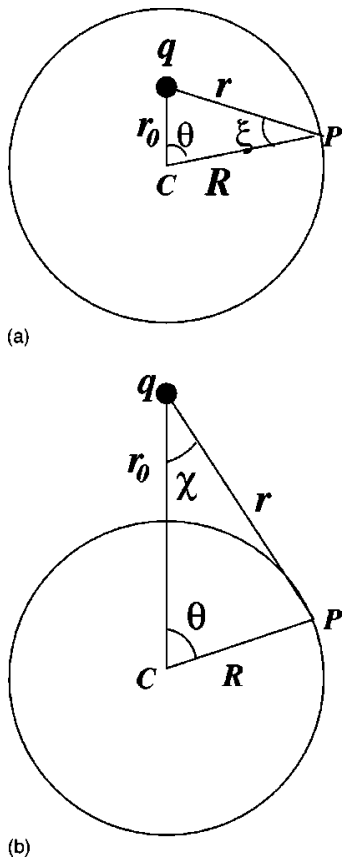

FIG. 7. Geometry of the charge and the shell in Sec. IV: (a) the charge is inside the shell; (b) the charge is outside the shell. $R$ is the shell's radius; $r_{0}$ is the distance between the charge $q$ and the shell's center $C . P$ is an arbitrary point on the shell. $r$ is the distance between the charge $q$ and point $P$. $\theta$ is the angle between lines $C q$ and $C P$; $\xi$ (in the upper figure) is the angle between lines $q P$ and $C P$, and $\chi$ (in the lower figure) is the angle between lines $q C$ and $q P$.

$$
\begin{aligned}
F^{\hat{z}} & =\frac{M}{4 \pi R^{2}} \int_{\text {shell }} d x^{2} \frac{q^{2}}{r^{3}} \cos (\theta+\xi) \\
& =\frac{q^{2} M}{2} \int_{-1}^{1} \frac{R \cos \theta-r_{0}}{\left(r_{0}^{2}+R^{2}-2 r_{0} R \cos \theta\right)^{2}} d(\cos \theta) \\
& =q^{2} \frac{G}{c^{2}} \frac{M}{R^{3}} \frac{1}{2 x_{0}}\left[\frac{1}{1-x_{0}^{2}}-\frac{1}{2 x_{0}} \ln \left(\frac{1+x_{0}}{1-x_{0}}\right)\right],
\end{aligned}
$$

where the angles $\theta$ and $\xi$ are defined in Fig. 7(a), and

$r=\sqrt{r_{0}^{2}+R^{2}-2 r_{0} R \cos \theta} \quad$ and $\quad \cos (\theta+\xi)=\frac{R \cos \theta-r_{0}}{r}$

We re-introduced Newton's constant $G$ and the speed of light $c$ in the last expression of Eq. (95). The self-force experienced by the charge is of the same magnitude but directs towards the center of the shell, which is exactly the same as Eq. (65).

When the charge is outside the shell, we have [Fig. 7(b)]

$$
\begin{aligned}
F^{\hat{z}} & =-\frac{M}{4 \pi R^{2}} \int_{\text {shell }} d x^{2} \frac{q^{2}}{r^{3}} \cos \chi \\
& =-\frac{q^{2} M}{2 r_{0}^{3}} \int_{-1}^{1} \frac{1-y_{0} \cos \theta}{\left(1+y_{0}^{2}-2 y_{0} \cos \theta\right)^{2}} d(\cos \theta) \\
& =-q^{2} \frac{G}{c^{2}} \frac{M}{2 r_{0}^{3}}\left[\frac{1}{1-y_{0}^{2}}+\frac{1}{2 y_{0}} \ln \left(\frac{1+y_{0}}{1-y_{0}}\right)\right],
\end{aligned}
$$

where we have used the expressions

$$
r=\sqrt{R^{2}+r_{0}^{2}-2 r_{0} R \cos \theta} \quad \text { and } \quad \cos \chi=\frac{r_{0}-R \cos \theta}{r}
$$

and re-introduced $G$ and $c$ in the last expression of Eq. (97). Hence, the self-force is $-F^{\hat{z}}$ (repel the shell), exactly the same as in Eq. (85).

We can carry out the above calculation also to the scalar charge case. Consider a scalar test charge at the origin of the coordinates. Recall that we are interested here in the selfforce, and not in the usual gravitational attraction due to the particle's mass. Hence, in what follows we ignore the mass of the scalar charge. Next, we write the coupled EinsteinKlein-Gordon equations,

$$
\begin{aligned}
& G_{\mu \nu}=8 \pi T_{\mu \nu} \\
& \square \Phi=-4 \pi \rho,
\end{aligned}
$$

where $\square$ denotes the covariant wave operator, and $\rho$ is the charge density given by Eq. (3) for a static charge at the origin, and look for a static, spherically symmetric solution. Here, $T_{\mu \nu}$ is the stress-energy tensor of a massless scalar field, which is given by

$$
T_{\mu \nu}=\frac{1}{4 \pi}\left(\Phi_{, \mu} \Phi_{, \nu}-\frac{1}{2} g_{\mu \nu} g^{\alpha \beta} \Phi_{, \alpha} \Phi_{, \beta}\right)
$$

The Einstein equations then reduce to

$$
\begin{aligned}
& R_{\mu \nu}=2 \Phi_{, \mu} \Phi_{, \nu} \\
& \square \Phi=-4 \pi \rho,
\end{aligned}
$$

whose solution is given by

$$
d s^{2}=-d t^{2}+\frac{d r^{2}}{1+\frac{q^{2}}{r^{2}}}+r^{2}\left(d \vartheta^{2}+\sin ^{2} \vartheta d \varphi^{2}\right)
$$




$$
\Phi_{, r}=-\frac{q}{r^{2}} \frac{1}{\sqrt{1+\frac{q^{2}}{r^{2}}}} \text { and } \Phi=\sinh ^{-1}(q / r)
$$

Hence the "gravitational force" experienced by a static point mass $\mu$ is $f^{\alpha}=-\mu D u^{\alpha} / D \tau=-\mu\left(u^{t}\right)^{2} \Gamma_{t t}^{\alpha}=0$, since $\Gamma_{t t}^{\alpha}=0$ for the metric (102). $D / D \tau$ here denotes covariant differentiation compatible with the metric (102). Thus we conclude that the self-force is zero to leading order in $\mu$. Consequently, after integration over the shell, the self-force is zero also to linear order in $M$. This result is in accord with our previous calculation that the self-force in the scalar charge case is a second post-Newtonian effect.

We can also make the following arguments for the direction of the self-force. For concreteness, consider the case of an electric charge inside the shell. The electric field lines near the charge have the usual distribution in space. However, outside the shell they are distorted due to the curvature of space. Specifically, the electric field lines are closer than what they would be if spacetime were flat. The reason is that the ratio of the circumference and the (proper) radius is smaller than $2 \pi$. When the charge is off the center of the sphere, the curvature effect is stronger outside the shell at the side closer to the charge. Because of the stress in the electrostatic field (one has to put in energy to squeeze electric field lines), this results in a force acting on the charge in the direction of the center of the sphere. An alternative viewpoint is the following. The electrostatic field is accompanied by a stress-energy tensor which gives rise to an effective metric which is similar to the Reissner-Nordström metric without the mass term. This metric induces repulsive (or anti-) gravity, which is the origin for the repulsive force acting on the charge. (The usual Reissner-Nordström geometry is attractive at large distances because of the mass term. It is, however, repulsive at short distances, where the mass term is small compared with the charge term in the metric. This happens, nevertheless, only deep inside the ReissnerNordström black hole, and is responsible for the phenomenon of gravitational bounce [29]. The occurrence of gravitational bounce in actuality is uncertain because of the innerhorizon instability of realistic black holes [30]. For recent reviews see $[31,32]$.) Consequently, the self-force on the charge directs toward the center of the shell.

\section{ACKNOWLEDGMENTS}

We thank Jeremy Heyl, Amos Ori, and Kip Thorne for invaluable discussions. L.M.B. wishes to thank the Technion Institute of Theoretical Physics, where part of this research was done, for hospitality. At Caltech this research was supported by NSF grants AST-9731698 and PHY-9900776 and by NASA grant NAG5-6840.
[1] A. I. Zel'nikov and V. P. Frolov, Zh. Éksp. Teor. Fiz. 82, 321 (1982) [Sov. Phys. JETP 55, 191 (1982)].

[2] A. G. Smith and C. M. Will, Phys. Rev. D 22, 1276 (1980).

[3] A. G. Wiseman, Phys. Rev. D 61, 084014 (2000).

[4] L. M. Burko, Class. Quantum Grav. 17, 227 (2000).

[5] B. Léauté and B. Linet, Class. Quantum Grav. 1, 55 (1984).

[6] B. Léauté and B. Linet, J. Phys. A 15, 1821 (1982).

[7] D. Lohiya, J. Phys. A 15, 1815 (1982).

[8] B. Linet and P. Teyssandier, Gen. Relativ. Gravit. 10, 313 (1979).

[9] B. Linet, Phys. Rev. D 33, 1833 (1986).

[10] A. G. Smith, in The Formation and Evolution of Cosmic Strings, edited by G. W. Gibbons, S. W. Hawking, and T. Vachaspati (Cambridge University Press, Cambridge, England, 1990).

[11] F. Ryan, Phys. Rev. D 56, 1845 (1997).

[12] W. G. Unruh, Proc. R. Soc. London A348, 447 (1976).

[13] B. Leaute and B. Linet, Gen. Relativ. Gravit. 17, 783 (1985).

[14] A. Ori (unpublished).

[15] L. Barack and A. Ori, Phys. Rev. D 61, 061502(R) (2000).

[16] T. C. Quinn and R. M. Wald, Phys. Rev. D 56, 3381 (1997).

[17] Y. Mino, M. Sasaki, and T. Tanaka, Phys. Rev. D 55, 3457 (1997).
[18] L. Barack, Phys. Rev. D 62, 084027 (2000).

[19] L. M. Burko, in Gravitational Waves: Third Edoardo Amaldi Conference, edited by S. Meshkov (AIP, New York, 2000) [gr-qc/9911089].

[20] L. M. Burko, Phys. Rev. Lett. 84, 4529 (2000).

[21] L. Barack and L. M. Burko, Phys. Rev. D 62, 084040 (2000).

[22] L. M. Burko, Am. J. Phys. 68, 456 (2000).

[23] C. O. Lousto, Phys. Rev. Lett. 84, 5251 (2000).

[24] A. G. Wiseman (unpublished).

[25] T. C. Quinn, Phys. Rev. D 62, 064029 (2000).

[26] W. Israel, Commun. Math. Phys. 8, 245 (1968).

[27] J. L. Anderson and J. M. Cohen, Astrophys. Space Sci. 9, 146 (1970).

[28] J. M. Cohen and R. M. Wald, J. Math. Phys. 12, 1845 (1971).

[29] I. D. Novikov, Zh. Éksp. Teor. Fiz., Pis'ma Red. 3, 223 (1966) [JETP Lett. 3, 142 (1966)]; V. De La Cruz and W. Israel, Nuovo Cimento A 51, 744 (1967).

[30] R. Penrose, in Battelle Rencontres, edited by C. DeWitt and J. A. Wheeler (Benjamin, New York, 1968).

[31] L. M. Burko and A. Ori, in Internal Structure of Black Holes and Spacetime Singularities, Volume XIII of the Annals of the Israel Physical Society, edited by L. M. Burko and A. Ori (Institute of Phsyics, Bristol, 1997).

[32] P. R. Brady, Prog. Theor. Phys. Suppl. 136, 29 (1999). 